\title{
Adaptive Multichannel Data Dissemination: Support of Dynamic Traffic Awareness and Push-Pull Time Balance
}

\author{
Chih-Lin Hu, Member, IEEE, and Ming-Syan Chen, Fellow, IEEE
}

\begin{abstract}
Data dissemination by the use of periodic broadcast is a viable scalable mechanism due to the explosion of information services in wireless networks. Many research works have exploited the data broadcast methodologies, however, upon the premise that the broadcast traffic is static on a single broadcast channel. In practice, the broadcast traffic changes dynamically and multiple broadcast channels can be utilized for efficient data dissemination. Accordingly, in this paper we devise an adaptive multichannel data-dissemination mechanism, including two components: the multichannel traffic-awareness and the deterministic balance search techniques. The multichannel traffic-awareness technique performs a cyclic estimation of dynamic access frequency distribution in response to traffic dynamics. With the measured estimation, the deterministic balance search technique adjusts channel allocation and item classification to further minimize and balance the push access and the pull response time. The experimental results show that the proposed mechanism is able to attain the result of channel partition and item classification very close to the optimum and, thus, minimize the mean access time in both push and pull channels simultaneously. Therefore, the reliability of broadcast contents is guaranteed.
\end{abstract}

Index Terms-Broadcast, data dissemination, hybrid data delivery, pull, push, traffic awareness, wireless network.

\section{INTRODUCTION}

$\mathbf{I}$ $\mathrm{N}$ TERMS of bandwidth capacity and information flow, the communication asymmetry imposes many constraints and challenges on the design and development of wireless information applications and services. Particularly, several networking systems, e.g., satellite networks, radio networks, and wireless links in cellular networks, have a larger downward bandwidth capacity than the upward one. In addition, the information flow can be asymmetric in instant messaging, personalized news distribution, advanced traffic information, and Web-surfing services, to name a few. Substantial researches have shown that the data broadcast/dissemination model is a viable solution to resolve the scalability problem and performance degradation, especially in the asymmetric communication environments [1], [5], [6], [31], [34].

Manuscript received May 15, 2003; revised March 8, 2004, July 6, 2004, and September 22, 2004. This work was supported in part by the National Science Council of Taiwan, R.O.C, under contract NSC93-2752-E-002-006-PAE. The review of this paper was coordinated by Bo Li.

C.-L. Hu is with the Research and Development Technology Division, Networking and Communications Business Group, BenQ Corporation, Taipei 114, Taiwan, R.O.C. (e-mail: charleshu@benq.com).

M.-S. Chen is with the Department of Electrical Engineering and Graduate Institute of Communication Engineering, National Taiwan University, Taipei 106, Taiwan, R.O.C. (e-mail: mschen@ @cc.ee.ntu.edu.tw).

Digital Object Identifier 10.1109/TVT.2004.841556
The advancement of data-broadcasting methodologies evolves three ramifications: push based, hybrid, and on-demand pull-based data-broadcast models. Originally, in the paradigm of push-based data broadcasting, the server applies a push broadcast program. Data items are pushed in a round-robin manner on the shared broadcast medium and accessed by clients without explicit requests [1], [19]. A broadcast cycle indicates a serial of interleaved data slots that the server delivers each push item at least once. However, the push-based delivery mode can result in an unacceptable access time when the number of push items is huge. The access time means the time interval from the moment at which a client requests an item until the moment that the item is downloaded completely. In contrast, the hybrid model makes the access time malleable [2], [8], [11], [15], [16], [28], [32]. Data items are classified as hot and cold items according to their access frequencies and item slots in a broadcast cycle are partitioned into the push and pull sets. The server pushes hot items on push slots periodically and delivers cold items on pull slots in the request-response manner. As for the on-demand (pull-based) model, often applied in a specific application domain, clients have to notify the server of on-demand access requests in advance of their broadcast downloading [3], [4], [30]. Then, the server later pushes data items responding to clients' access interests.

The push-based and hybrid data-broadcast models, however, have no fundamental support of a dynamic traffic-awareness mechanism. Particularly, a server does not perceive dynamic changes of access commonality, client population, and workload because a client, in a sense, is passive and does not notify the server of its broadcast access. This phenomenon challenges the server to conform the broadcast contents to clients' access interests. Notwithstanding, many researches have advanced the data-broadcast methodology [6], [31], although their efforts did not offer adaptability against traffic dynamics since they assumed that either the broadcast traffic is static or that the knowledge of the previous traffic patterns is available.

To this end, a few traffic-awareness techniques [8], [10], [16], [14], [28], [36] are presented. The probing technique is first applied to calculate the access statistics, but may induce a scalability problem [28]. A partial probe or sampling technique is used to alleviate the performance degradation, but needs a longer calculation time to obtain the complete access statistics [10]. In [8] and [16], the feedback/piggyback technique appends the access statistics information into the clients' pull requests; however, at the cost of having extra representations of feedback information [21]. Moreover, the convention of 
push access in the client side will be changed, since a passive client must actively report the feedback information. In [36], a statistic maximum-likelihood estimation method formulates the broadcast miss as a binomial distribution, which is essentially dedicated to estimate the effectiveness of static scheduling. Comparatively, a selective deferment and reflection (SDR) technique in [14] estimates dynamic access frequency distribution on the single-channel data broadcast model. In addition, the work in [24] proposes a single-channel adaptation algorithm that can approximate the optimal system response time by maintaining a certain level of on-demand request arrival rate without knowing the exact request arrival rates, but only the relative popularity of data items.

The recent advent of the multichannel data broadcast has attracted many researchers' attention [17], [23], [25]-[27], [35]. As the downward bandwidth is composed of multiple channels, channels are allocated on discontiguous spectrums and cannot be coalesced into a shared channel. There are several reasons for disseminating data over multiple channels instead of sending them over a dedicated fast channel. Some are based on the physical constraints of communication architectures. For example, clients may have heterogenous communication capabilities, limiting the feasibility of single-channel transmission [9]. The reconfiguration of adjacent cells can result in a multichannel environment. In addition, the application-based use of multiple channels is considered for application scalability and fault tolerance. Channels can be combined or coordinated to offer variable quality of service to clients; they can also be used to ensure the efficacy in the hybrid push-pull systems. Bandwidth conservation is yet another use. For example, some applications may require only a fraction of total available bandwidth, so leftover bandwidth can be used by other applications.

Therefore, the multichannel data broadcast model has different channel utilization and granularity of item replacement/switch [23], [25], as compared to the single-channel model. Explicitly, a channel switch from the push/pull mode to the other involves a number of items replaced/switched into the pull/push set as opposed to one item switch in the single-channel data broadcast model [2], [8], [11], [15], [28]. Several papers have explored the multichannel data broadcast model. The work in [23] presents an analytical study of the multichannel partition. In [26], the work designs the multichannel caching scheme. In [18], [25] the broadcast-disk-like [1] programs on multiple channels are dynamically generated to improve energy saving. In [12], the work presents a fair queueing method used for the static optimization of single-/multichannel broadcast scheduling, in accordance with predetermined access patterns. In [17], the work optimizes the broadcast schedule on multiple channels for a complex query retrieving multiple dependent data items. The work in [35] proposes several indexing schemes for a multichannel broadcast program. However, same as most studies in single-channel model, these papers are not adaptive against unpredictable traffic dynamics.

In this paper, we examine the issues of adaptively disseminating broadcast contents on multiple broadcast channels in the hybrid data broadcast environment. The design of a reliable multichannel data-dissemination mechanism is a critical challenge, while the data broadcast traffic has the nature of dynamic changes. Although a few techniques, e.g., the feedback/piggyback, probing, sampling and SDR techniques, have been presented to perceive dynamic traffic changes, they are dedicated to the single-channel model. Extended or new techniques are desired to efficiently measure various factors of traffic dynamics in multichannel environments. Furthermore, we investigate that the hybrid data delivery must adjust channel allocation and item classification based on the obtained traffic measure. In doing so, the information server will, thus, be able to maintain the appropriateness of the broadcast contents and balance the push and the pull access time.

This paper devises an adaptive multichannel data-dissemination mechanism, called AMD for brevity, in the multichannel data-broadcast environment. The AMD mechanism includes two basic components: the multichannel traffic-awareness (MCTA) and the deterministic balance search (DBS) techniques. The MCTA technique exploits the nature of client impatience [20] to periodically estimate the dynamic access frequency distribution in multiple broadcast channels. While accessing broadcast items, clients have limited patience. With the measure of impatient requests of all broadcast items within a broadcast cycle time, the server reflectively estimates the access frequency distribution among all items in all broadcast channels. According to the cyclic estimation by the MCTA technique, the DBS technique further deals with the adaptation of item classification and channel allocation and, thus, is able to simultaneously minimize and balance the push access time and the pull response time. As a result, the AMD mechanism employs the MCTA and DBS techniques repeatedly per broadcast cycle to sustain traffic dynamics. Therefore, the reliability of broadcast contents is guaranteed.

For the implementation, the MCTA technique can be elegantly applied to the hybrid data broadcast model. The DBS technique is of low complexity and can be executed online without complicating the data-broadcasting system. Extensive simulations are conducted in this paper. Experimental results show that the AMD mechanism is able to attain a push-pull time balance very close to the optimum, while various traffic conditions are examined. Consequently, the AMD mechanism with all-inclusive traffic factors can provide a foundation for the adaptation of broadcast scheduling, indexing, and the hybrid data-delivery as compared to the previous one, which resolves a static optimization problem in a single-channel data broadcast model.

The rest of this paper is organized as follows. Section II mentions the system modeling and notation. Section III devises the MCTA technique. The design of the DBS technique is given in Section IV and Section V demonstrates the performance evaluation. This paper concludes with Section VI.

\section{SySTEM MODELING AND NOTATION}

Fig. 1 illustrates the environment in which we design the AMD mechanism. A wireless information server has a broadcast database $D=\left\{d_{1}, d_{2}, \ldots, d_{m}\right\}$ that contains $m$ items of the same size $s$. Suppose that the slotted time model is employed. It takes one time slot to broadcast each item. Therefore, the terminologies of time and data slots are used inter- 


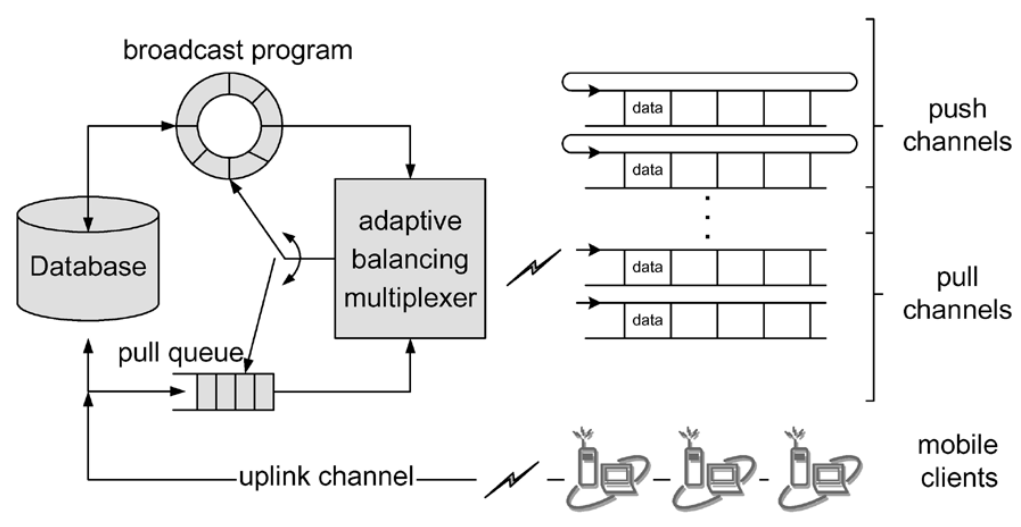

Fig. 1. Adaptive multichannel data-dissemination mechanism.

TABLE I

PARAMETERS USED IN THE AMD MECHANISM

\begin{tabular}{cl}
\hline notation & \multicolumn{1}{c}{ meaning } \\
\hline$m$ & the number of items in the broadcast database $D$ in the server, i.e., $|D|$ \\
$d_{i}^{x}$ & an item in the $i$-th slot of the $x$-th channel in a broadcast cycle \\
$U^{h}$ & the push item set including hot (popular) items \\
$U^{c}$ & the pull item set including cold (unpopular) items \\
$\lambda_{i}(j)$ & the access rate for the item $d_{i}^{x}$ in the $j$-th time unit \\
$\lambda_{i}^{x}$ & the aggregate of access rates for item $d_{i}^{x}$ in a broadcast cycle \\
$\gamma_{i}^{x}$ & the number of impatient requests for item $d_{i}^{x}$ in a broadcast cycle \\
$P_{x}$ & the coordinative broadcast program in the $x$-th push channel \\
$P$ & the compound broadcast program \\
$L$ & the length of a coordinative broadcast cycle \\
$\omega$ & mean client patience time \\
$c_{\text {push }}$ & the number of push channels for broadcasting hot items in $U^{h}$ \\
$c_{\text {pull }}$ & the number of pull channels for delivering cold items in $U^{c}$ \\
$\Phi_{i}^{x}(x)$ & the estimated access frequency distribution of $P_{x}$ with the reflective base $d_{i}^{x}$ \\
$\Psi^{x}(x)$ & the real access frequency distribution of $P_{x}$ \\
$\Phi_{\text {push }}^{x}(x)$ & the estimated access frequency distribution among all hot items in $P_{x}$ \\
$\Phi_{\text {pull }}(x)$ & the pull access frequency distribution among all cold items in a broadcast cycle \\
$\Phi_{\text {push }}(x)$ & the push access frequency distribution among all hot items in $P$ in a broadcast cycle \\
$\Psi(x)$ & the real access frequency distribution \\
\hline
\end{tabular}

changeably, where there is no ambiguity. Let the access rate of each item in a time unit $t_{j}$, denoted as $\lambda_{i}(j)$, form a Poisson process with an aggregate arrival rate $\lambda=\sum_{i=1}^{m} \lambda_{i}(j)$ and $\lambda_{1}(j) \geq \lambda_{2}(j) \geq \cdots \geq \lambda_{m}(j)$. All items in $D$ are classified into a push item set $U^{h}=\left\{d_{1}, d_{2}, \ldots, d_{k}\right\}$ and a pull item set $U^{c}=\left\{d_{k+1}, \ldots, d_{m}\right\}$ according to the relative access rates. It is noted that $d_{k}$ is the "optimal cutoff item" suggested by the AMD mechanism. Suppose that the downward bandwidth consists of $c$ channels of equal bandwidth. The server allocates $c_{\text {push }}$ channels for periodically broadcasting items in $U^{h}$. Other $c_{\text {pull }}=c-c_{\text {push }}$ channels carry cold items in the request-response manner.

Definition 1: Given that $P_{x}$ is a coordinative broadcast program $\left\{d_{1}^{x}, d_{2}^{x}, \ldots, d_{L}^{x}\right\}$ in the $x$ th push channel within a broadcast cycle $L$, a compound broadcast program $\mathcal{P}$ is a sequential union $\cup_{x=1}^{c_{\text {push }}} P_{x}=\mathcal{P}_{1} \cup \cdots \cup \mathcal{P}_{c_{\text {push }}}$.

To maintain the generality, a flat broadcast schedule policy is applied to provide a fundamental basis in comparison with others. Specifically, hot items in $U^{h}$ are arranged into push channels in descending order of relative access frequencies without item duplication and dummy slots. As defined in Definition 1, a compound broadcast program $P$ is the union of coordinative broadcast programs in push channels. Hence, a broadcast cycle
$L$ is equal to the number of slots in each $P_{x}$. For convenience, we term $d_{i}^{x}$ as an item scheduled in the $i$ th slot of $P_{x}$. In addition, we assume that the index of $\mathcal{P}$ can be transmitted to the clients ahead of the push data either through the push channels or a distinct channel, so that a client can decide to access an item by the pull/push mode. A client who has interest in $d_{i}^{x}$ can submit an impatient pull request for $d_{i}^{x}$ after its waiting time has exceeded its mean patience $w$. The amount of impatient pull requests for $d_{i}^{x}$ is denoted as $\gamma_{i}^{x}$ within a cycle time. Note that, in practice, the total number of the accumulative impatient requests is independent of the broadcast index. Guided by the index, a client can be in the active/passive mode to reduce the power and energy consumption, but it remains at waiting for the interested item under its patience. Table I lists the notation used in this paper. The assumptions adopted in this study are:

- length of a broadcast cycle is not fixed;

- each item is self-identified and read-only;

- downlink channel is in either the pull or push mode and can be alternatively switched to complement other channels;

- policy to the channel allocation is the fair balance between the push access and the pull response time;

- policy to the item classification is the relative access rate. 


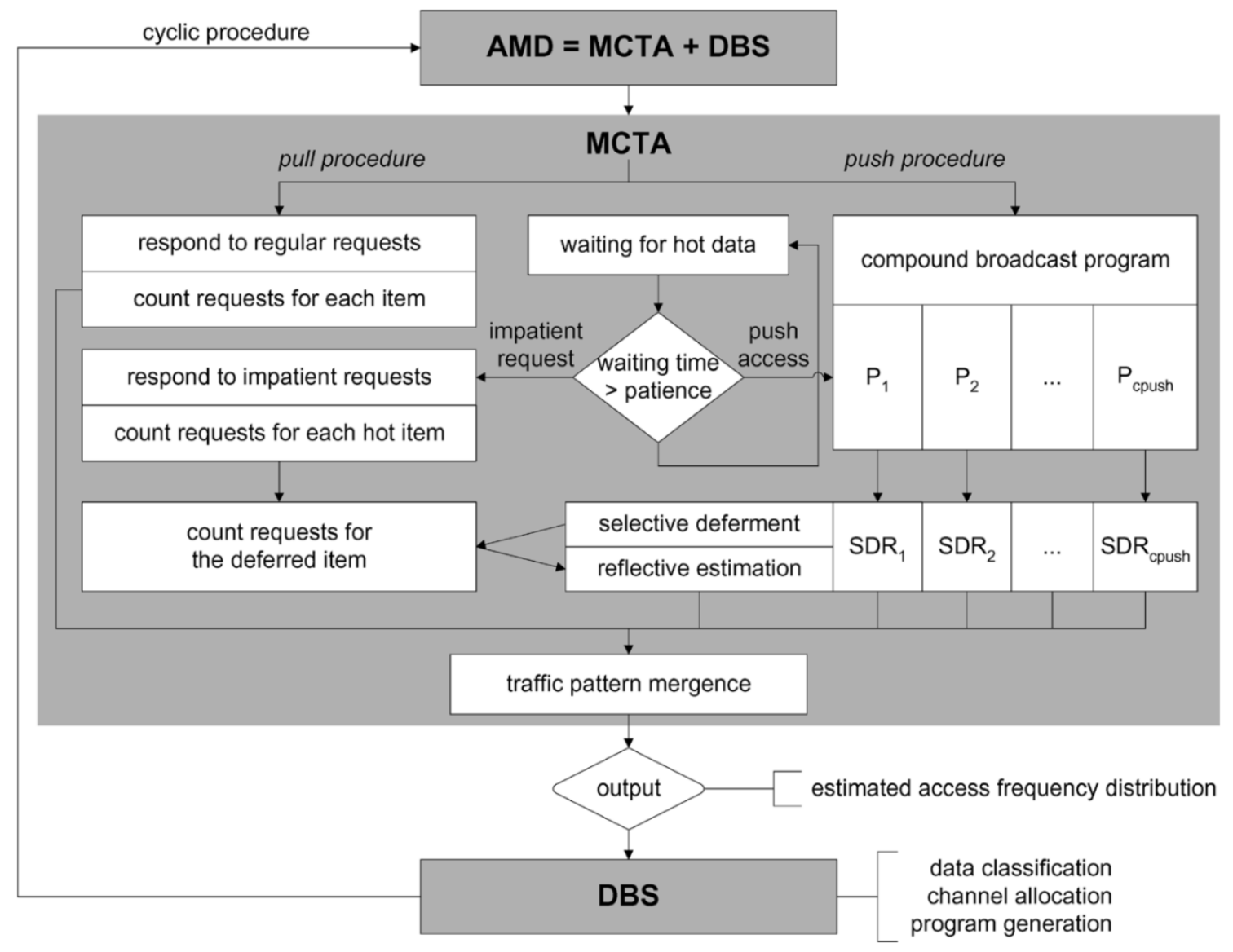

Fig. 2. Flowchart of the MCTA mechanism.

Specifically, the design in this paper does not confine clients' access behavior. A client can simultaneously access multiple data items from multiple channels. Moreover, a client is allowed to access another push item or to submit a new pull request before its latest pull request, i.e., a pending request, will be satisfied. However, some studies assumed that a client can only have a pending request at a time so that the access workload is controllable and proportional to the number of clients. For the sake of generality, our study need not know the number of clients, which is in essence a predetermined traffic factor in some works [16], [32]. This model is similar to the data broadcast environments in [2], [8], [28], but having a graceful extension for the multichannel data broadcast environments.

\section{Multichannel Traffic Awareness}

Section III-A gives the design abstraction and Section III-B describes the calculation of the pull access frequency distribution. The estimation of the push access frequency distribution is presented in Section III-C.

\section{A. Design Abstraction}

Fig. 2 instantiates the flowchart of the MCTA procedure that includes two procedures: pull and push. The pull procedure calculates the exact number of pull requests for each cold item and the push procedure estimates the access frequency distribution of all hot items broadcast on all push channels. Note that the server maintains a compound broadcast program that consists of multiple coordinative broadcast programs on different push channels. Clients have limited patience in waiting hot items on push channels. On each push channel, the SDR technique [14] is applied to estimate the push access distribution of each coordinative program. Particularly, multiple coordinative programs have the same length of the broadcast cycle. The server makes the single-item broadcast miss synchronously, for a cycle time, on each push channel with a specific reflective base, i.e., the missed item and, thus, simultaneously obtains the reflective estimations of all coordinative programs. Then, the mergence of multiple estimations generates the access frequency distribution of the compound broadcast program. Finally, the combination of the pull and push access frequency distributions results in the overall access frequency distribution among all cold and hot items. Hence, the server perceives dynamic traffic changes. In addition, the cyclic estimation of the access frequency distribution, by means of the MCTA technique, provides the primary information for the DBS technique to further adjust item classification and channel allocation and balance the push access and pull response time, as will be devised in the next section.

Note that the MCTA procedure can be deemed a special case of the probing or sample technique, since the server employs a single-item broadcast miss for a cycle time to disclose the exact access frequency of the reflective base. According to the reflective base, the MCTA technique is able to derive the access 


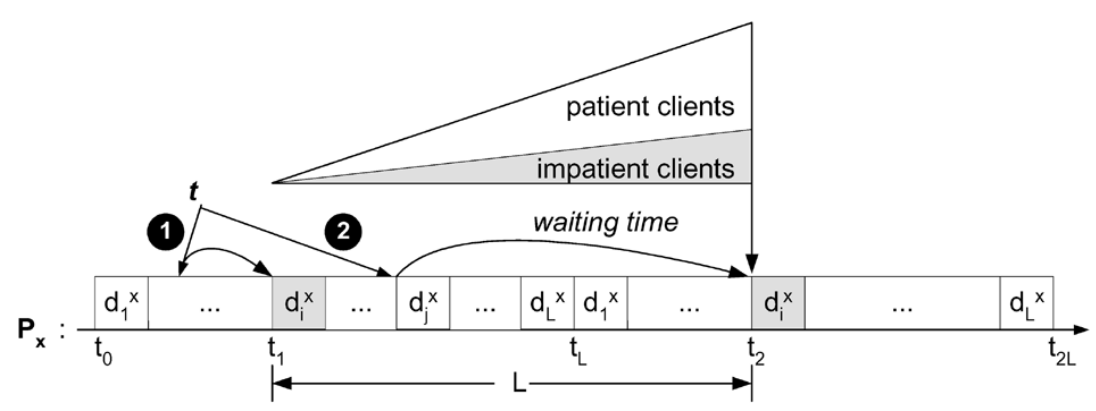

Fig. 3. Push access estimation in $P_{x}$.

frequencies of other push items in the coordinative program. In contrast, the probing/sampling technique must apply a series of broadcast misses to have access frequencies of all push items in each push channel.

\section{B. Pull Access Frequency Distribution}

In the hybrid data delivery model, the server has the statistic of exact access frequencies of all cold items. During the time interval of a broadcast cycle $L$, the server counts the total access frequency $\lambda_{i}$ of each cold item $d_{i}$ in $U^{c}$. The mean access frequency of $d_{i}$ is $\overline{\lambda_{i}}=\left(\lambda_{i}\right) /(L)$ for $k+1 \leq i \leq m$. Then, the mean pull access frequency distribution $\Phi_{\text {pull }}(x)$ among pull items can be expressed as

$$
\Phi_{\text {pull }}(x)=\left\langle\overline{\lambda_{k+1}}, \overline{\lambda_{k+2}}, \ldots, \overline{\lambda_{m}}\right\rangle
$$

\section{Push Access Frequency Distribution}

The hot items $U^{h}$ in the compound broadcast program $P$ is the superset of all items in $c_{\text {push }}$ coordinative programs $P_{x} \mathrm{~s}$. The push access frequency distribution $\Phi_{\text {push }}(x)$ merges all of the estimated distributions $\Phi_{\text {push }}^{x}(x) \mathrm{s}$ of $P_{x}$ s on push channels. Without a loss of generality, we assume that clients have the same access behavior on different channels. Thus, the server obtains $\Phi_{\text {push }}^{x}(x)$ s simultaneously and generates $\Phi_{\text {push }}(x)$.

1) Estimation of Coordinative Program: The estimation procedure on each $P_{x}$ has three phases: 1) client impatience modeling; 2) selective deferment and reflective estimation; and 3) estimation enhancement with the trigonometric tuning method. We modify the SDR technique [14] appropriately for the use and representation in the multichannel data broadcast context. The modified parts are recapitulated as follows.

Client Impatience Modeling: Suppose a client arrives at the $j$ th slot and has interest in $d_{i}^{x}$ on the coordinative program $P_{x}$. Fig. 3 illustrates two cases in regard to a client's push access. In case 1 , when the item has not been broadcast in the current cycle, the client has to wait $i-j-(1 / 2)$. However, in case 2, when the item has been delivered in advance of the client's push access in this cycle, the waiting time is $L+i-j-(1 / 2)$ slots. Let a random variable $x$ present a client's patience in an exponential distribution with a mean patience $\omega$ [20]. The probability density function of $x$ is given by $f_{\gamma}(x)=(1 / \omega) e^{-(1 / \omega) x}$ as $0 \leq x<\infty$, and the distribution function is $F_{\gamma}(x)=1-e^{-(1 / \omega) x}$ as $0 \leq x<\infty$. Accordingly, the probability that a client will generate an impatient pull request for $d_{i}^{x}$ is

$$
\begin{aligned}
\mathcal{F}_{\gamma}(i, j) & \\
\quad= & \begin{cases}\int_{0}^{i-j-\frac{1}{2}} f_{\gamma}(x) d x=1-e^{-\frac{1}{\omega}\left(i-j-\frac{1}{2}\right)}, & i>j \\
\int_{0}^{L+i-j-\frac{1}{2}} f_{\gamma}(x) d x=1-e^{-\frac{1}{\omega}\left(L+i-j-\frac{1}{2}\right)}, & i \leq j\end{cases}
\end{aligned}
$$

where $1 \leq i, j \leq L$. Hence, the aggregate of impatient pull requests for $d_{i}^{x}$ in a cycle time is $\gamma_{i}^{x}=\sum_{1<j \leq L} \lambda_{i}^{x}(j) \cdot \mathcal{F}_{\gamma}(i, j)$ where $\lambda_{i}^{x}(j)$ is the access rate of $d_{i}^{x}$ in the $j$ th slot. Observe that if every client has an equal mean patience $\omega$, the value of $\sum_{1 \leq j \leq L} \mathcal{F}_{\gamma}(i, j)$ is the same for each push item. We then have

$$
\gamma_{i}^{x}=\sum_{1 \leq j \leq L} \overline{\lambda_{i}^{x}} \cdot \mathcal{F}_{\gamma}(i, j)=\overline{\lambda_{i}^{x}} \cdot\left(L-e^{-\frac{L^{2}}{2 \omega}}\right) .
$$

Since $\left(L-e^{-\left(L^{2}\right) /(2 \omega)}\right)$ is constant, we have the ratio equation

$$
\gamma_{1}^{x}: \vdots \gamma_{i}^{x}: \vdots \gamma_{k}^{x}=\overline{\lambda_{1}^{x}}:: \overline{\lambda_{i}^{x}}:: \vdots \overline{\lambda_{k}^{x}}
$$

Selective Deferment and Reflective Estimation: To solve the values in (4), the server intentionally selects a single item $d_{i}^{x}$ in $P_{x}$ and temporarily defers its broadcast for a cycle time. The selective deferment compels the clients to disclose their interests in $d_{i}^{x}$. Fig. 3 depicts the selective deferment and reflective estimation. In the time interval $\left[t_{1}, t_{2}\right]$, the server will receive $\gamma_{i}^{x}=\sum_{1 \leq j \leq L} \lambda_{i}^{x}(j) \cdot \mathcal{F}_{\gamma}(i, j)$ impatient requests for $d_{i}^{x}$. Moreover, some patient clients will submit regular pull requests for $d_{i}^{x}$ when they find that $d_{i}^{x}$ is absent at $t_{2}$. Then, the server can obtain the exact access frequency $\lambda_{i}^{x}$ by adding $\gamma_{i}^{x}$ and the number of the regular pull requests for $d_{i}^{x}$. Likewise, other impatient frequencies $\left\langle\gamma_{1}^{x}, \gamma_{2}^{x}, \ldots, \gamma_{k}^{x}\right\rangle$ are available. Note that with a reflective base $\lambda_{i}^{x}$ in (4), the server is able to reflectively estimate the access frequency of each push item as

$$
\overline{\lambda_{j}^{x}}=\frac{\gamma_{j}^{x}}{\gamma_{i}^{x}} \cdot \frac{\lambda_{i}^{x}}{L}=\frac{\gamma_{j}^{x}}{\gamma_{i}^{x}} \cdot \overline{\lambda_{i}^{x}}, \quad 1 \leq j \leq k .
$$

By (4) and (5), the server can obtain the dynamic access frequency distribution $\Phi_{\text {push }}^{x}(x)$ of each $P_{x}$ in a cycle time as

$$
\begin{aligned}
\Phi_{\mathrm{push}}^{x}(x)=\left\langle\frac{\gamma_{1}^{x}}{\gamma_{i}^{x}} \cdot \overline{\lambda_{i}^{x}}, \ldots, \frac{\gamma_{i-1}^{x}}{\gamma_{i}^{x}} \cdot \overline{\lambda_{i}^{x}}, \overline{\lambda_{i}^{x}}, \frac{\gamma_{i+1}^{x}}{\gamma_{i}^{x}}\right. \\
\left.\cdot \overline{\lambda_{i}^{x}}, \ldots, \frac{\gamma_{k}^{x}}{\gamma_{i}^{x}} \cdot \overline{\lambda_{i}^{x}}\right\rangle
\end{aligned}
$$


Estimation Enhancement With Trigonometric Tuning: The MCTA technique has the theoretical and quantitative analyses inherent in the SDR technique. Some analytical results extracted from [14] are used to devise the trigonometric tuning method for estimation enhancement.

Theorem 1: $\Phi_{\text {push }}^{x}(x)$ with a reflective base $d_{i}^{x}$ has at least a cross with $\Psi_{\text {push }}^{x}(x)$ at $\Phi_{\text {push }}^{x}(i)=\Psi_{\text {push }}^{x}(i)$.

Proof: This theorem follows from the fact that the server only perceives the exact number of push accesses for $d_{i}^{x}$.

Theorem 2: With a reflective base $d_{i}^{x}$ whose $\left(\lambda_{i}^{x}\right) /\left(\gamma_{i}^{x}\right)$ is the smallest, we have $\lambda_{i}^{x}=\Phi_{\text {push }}^{x}(i) \lesssim \Psi_{\text {push }}^{x}(i)$ if $\lambda_{i}$ is continuously increasing or $\lambda_{i}^{x}=\Phi_{\text {push }}^{x}(i) \gtrsim \Psi_{\text {push }}^{x}(i)$ if $\lambda_{i}$ is continuously decreasing.

Proof: In accordance with (5), when the workload increases continuously, using the item $d_{i}^{x}$ of the smallest $\left(\lambda_{i}^{x}\right) /\left(\gamma_{i}^{x}\right)$ as the reflective base results in the estimated values of other push items in $P_{x}$ to be lower than their real values. Therefore, we have $\Phi_{\text {push }}^{x}(i) \lesssim \Psi_{\text {push }}^{x}(i)$. Contrarily, this item $d_{i}^{x}$ has the largest value of $\left(\lambda_{i}^{x}\right) /\left(\gamma_{i}^{x}\right)$ when the workload decreases continuously so that other push items in $P_{x}$ have the estimated values of $\Phi_{\text {push }}^{x}(i)$ to be higher than their real values. As a result, we have $\Phi_{\text {push }}^{x}(i) \gtrsim \Psi_{\text {push }}^{x}(i)$.

Property 1: Given a flat $P_{x}$ scheduled in order of access frequency, the item of the smallest $\left(\lambda_{i}^{x}\right) /\left(\gamma_{i}^{x}\right)$ is either $d_{\lceil L / 2\rceil}$ or an item close to $d_{\lceil L / 2\rceil}$.

Property 2: Given a flat $P_{x}$ scheduled randomly, the server can sort the items by their $\gamma_{i}^{x}$ s and take $d_{\lceil L / 2\rceil}$ as the reflective base if $P_{x}$ is scheduled randomly.

Property 3: Given an increasing/decreasing workload with a static access pattern, the average of $\Phi_{1}^{x}(x)$ and $\Phi_{\lceil L / 2\rceil}^{x}(x)$ has two cross points with $\Psi^{x}(x)$ if $P_{x}$ is in order of the access frequency.

Accordingly, we further devise a tuning method based on the semi-monotonic feature of trigonometric functions to enhance the estimation. Given a $P_{x}$ of $L$ item slots, each slot is subsequently mapped to an angle range of $[0, \pi]$ in radians. For instance, the angle of $d_{1}^{x}$ is 0 , the angle of $d_{\lceil L / 2\rceil}^{x}$ is $(\pi / 2)$, and other $d_{i}^{x}$ has its angle $\delta_{i}$ as $i \cdot(\pi / L)$ where $1 \leq i \leq L$. Then, we have a tuned distribution $\Phi_{\Delta}^{x}(x)$ as

$$
\Phi_{\Delta}^{x}(x)=\cos ^{2} \delta_{x} \cdot \Phi_{1}^{x}(x)+\sin ^{2} \delta_{x} \cdot \Phi_{\lceil L / 2\rceil}^{x}(x)
$$

where $\cos ^{2} \delta_{x}+\sin ^{2} \delta_{x}=1$. Because $\Phi_{\lceil L / 2\rceil}^{x}(x) \operatorname{crosses} \Psi^{x}(x)$ at $d_{\lceil L / 2\rceil}$, we apply a sine function to $\Phi_{\lceil L / 2\rceil}^{x}(x)$. Likewise, we apply a cosine function to $\Phi_{1}^{x}(x)$, which crosses $\Psi^{x}(x)$ at $d_{1}$. Hence, we can tune the estimation in the range between $\Phi_{1}^{x}(x)$ and $\Phi_{\lceil L / 2\rceil}^{x}(x)$ with two cross points at the point $\delta_{x}=(\pi / 2)$, where $\Phi_{\Delta}^{x}(L / 2)=\Phi_{\lceil L / 2\rceil}^{x}(L / 2)=\Psi^{x}(L / 2)$ and at the point $\delta_{x}=0$ with $\Phi_{\Delta}^{x}(1)=\Phi_{1}^{x}(1)$.

2) Estimation of Compound Program: In the end of each broadcast cycle, the server is able to merge $\Phi_{\text {push }}^{x}(x) \mathrm{s}$ of $P_{x} \mathrm{~s}$ from $c_{\text {push }}$ channels and generate a push access frequency distribution $\Phi_{\text {push }}(x)$ of $P$. Then, the combination of $\Phi_{\text {push }}(x)$ and $\Phi_{\text {pull }}(x)$ results in an overall access frequency distribution $\Phi(x)$ for all items in the broadcast database.

Push Mergence: In a divide-and-consquer strategy, the task of estimating $\lambda_{i}^{x}$ s of push items is recursively divided into $c_{\text {push }}$ smaller tasks, which include $L$ push items and are solved simultaneously. The conquering phase integrates the answers together. Given with $\Phi_{\text {push }}^{1}(x), \ldots, \Phi_{\text {push }}^{c_{\text {push }}}(x)$, the push mergence achieves a $\Phi_{\text {push }}(x)$ with some sorting algorithm [33].

Combined Mergence: With two sorted lists, $\Phi_{\text {pull }}(x)$ and $\Phi_{\text {push }}(x)$, the sorting algorithm is employed to generate a sorted overall access frequency distribution $\Phi(x)$.

Running-Time Analysis: When the order of $\Phi_{\text {push }}^{x}(x)$ is sorted, the push mergence has its average running time $O\left(m \log c_{\text {push }}\right)$. Otherwise, the push mergence has its average running time $O(m \log m)$. In addition, as for the running time of the combined mergence, the time to merge $\Phi_{\text {pull }}(x)$ and $\Phi_{\text {push }}(x)$ is linear to the number of items, i.e., $\Theta(m)$, because $\Phi_{\text {pull }}(x)$ and $\Phi_{\text {push }}(x)$ are sorted.

\section{Deterministic Balance Search}

In light of the time-measure formulas in Section IV-A, Section IV-B devises the deterministic balance search that is used to balance the push access time and the pull response time for a hybrid data delivery in a multichannel model.

\section{A. Time-Measure Formulation}

A random client's item access to $d_{i}^{x}$ falls into one of three cases: 1) $d_{i}^{x}$ is downloaded from some push channel when the waiting time is smaller than a client's patience $w$;2) $d_{i}^{x}$ is received indirectly by an impatient pull request because the waiting time exceeds $w$; or 3) $d_{i}^{x}$ is a cold item and accessed by a regular pull request. Accordingly, we express the time-measure formulas of the mean push access time and the mean pull response time as follows. Note that it is assumed in this study that multiple channels are coalesced, not merged, to serve the data broadcasting since channels can be allocated in different spectrum.

1) Mean Push Access Time: Given that $c_{\text {push }}$ channels are used to deliver $k$ push items in $U^{h}$, each push channel needs to transmit $\left(k / c_{\text {push }}\right)$ items in a cycle time. Let each channel have the bandwidth capacity of $b \mathrm{~B}$ and each item have the equal size of $s$ B. With a channel service rate $\mu=(b / s)$ items, according to the linear expectation property, the mean push access time $\overline{t_{\text {push }}}$ is

$$
\overline{t_{\mathrm{push}}}=\frac{1}{2} \cdot \frac{s k}{b c_{\mathrm{push}}}+\frac{s}{b}=\frac{k}{2 \mu c_{\mathrm{push}}}+\frac{1}{\mu} .
$$

2) Mean Pull Response Time: The pull requests comprise the regular pull requests for cold items and impatient pull requests for push items. Let both the arrival rates of the regular pull requests and the impatient pull requests be the Poisson distributions with parameters $\lambda_{\text {pull }}=\sum_{i=k+1}^{m} \lambda_{i}$ and $\sum_{i=1}^{k} \gamma_{i}^{x}$, respectively. We note that the arrival rate of total pull requests also follows the Poisson process. Specifically, same as some prior works on data broadcasting [4], [8], [23], [29], [34], [36], we assume that clients are assumed to access push items according to a Poisson process and that the client patience has an exponential distribution [20], also mentioned in Section III-B. In light of the probability-generating technique [13], the sum of two independent random variables, i.e., $\lambda_{\text {pull }}$ and $\sum_{i=1}^{k} \gamma_{i}^{x}$, also has the Poisson distribution with a parameter $\lambda_{\text {pull }}^{\prime}=\lambda_{\text {pull }}+\sum_{i=1}^{k} \gamma_{i}^{x}$. On the other hand, we assume that the size of each item is fixed 
and the same as others. The service time of broadcasting an item is, thus, deterministic. Accordingly, we employ an $M / D / m$ queueing model to estimate the mean pull response time on the multiple channels. The traffic intensity (or server utilization) is given by $\rho=($ arrival rate $) /($ service rate $)=\left(\lambda_{\text {pull }}^{\prime}\right) /\left(\mu c_{\text {pull }}\right)$, where $\mu$ is the service rate of each pull channel and $c_{\text {pull }}$ pull channels are available. Under a stable condition $\rho<1$ [22], the mean pull response time is given as

$$
\overline{t_{\text {pull }}}=\frac{1}{\mu}+\overline{W_{M / D / m}}=\frac{1}{\mu}+\frac{1}{2} \cdot \frac{1}{n c_{D m}} \cdot \overline{W_{M / M / m}}
$$

where $\overline{W_{M / D / m}}$ is the mean waiting time in the $M / D / m$ system, $n c_{D m}$ is the factor of Cosmetatos approximation, and $\overline{W_{M / M / m}}$ is the mean waiting time in the $M / M / m$ system. The equations of $n c_{D m}$ and $\overline{W_{M / M / m}}$ are given in [7]

$$
\begin{aligned}
n c_{D m}= & \left(1+(1-\rho)(m-1) \frac{\sqrt{4+5 m}-2}{16 \rho m}\right)^{-1} \\
\overline{W_{M / M / m}}= & \frac{1}{\left(\mu c_{\text {pull }}-\lambda_{\text {pull }}^{\prime}\right)} \cdot \frac{\left(\rho c_{\text {pull }}\right)^{c_{\text {pull }}}}{c_{\text {pull }} !(1-\rho)} \\
& \times\left(\sum_{k=0}^{c_{\text {pull }}-1} \frac{\left(\rho c_{\text {pull }}\right)^{k}}{k !}+\frac{\left(\rho c_{\text {pull }}\right)^{c_{\text {pull }}}}{c_{\text {pull }} !(1-\rho)}\right)^{-1} .
\end{aligned}
$$

Hence, with the above equations, we have the mean pull response time from (9).

\section{B. Deterministic Balance Search}

This section describes several heuristic observations and the DBS procedure for the adaptation of a hybrid data delivery in a multichannel environment.

1) Heuristics: To clarify the substance, several observations are made for the design of the DBS procedure.

- Observation 1 (item classification): In general, the relative access frequency is considered the policy of item classification. Since the estimated access frequency distribution $\Phi(x)$ is able to offer the discrimination with respect to access commonality, the phase of item classification should look for a proper cutoff point (at the $k$ th position) splitting the list into the push and the pull lists.

- Observation 2 (channel allocation): To efficiently deliver the respective items in the push and pull sets, the server must allocate the push and pull channels appropriately. Let all channels have $L$ slots equally. The server takes $c_{\text {push }}=$ $\lceil k / L\rceil$ push channels to broadcast $k$ items in $U^{h}$ and the pull items in $U^{c}$ are assigned to other $c_{\text {pull }}=c-c_{\text {push }}$ channels. Note that item slots in the same channel are inseparable. That said, the granularity in a multichannel model is a channel switch involving $L$ item slots.

- Observation 3 (adaptation of fairness and balance): The server must be able to manipulate the cutoff point so that the result of channel allocation and item classification approximates to the optimum of fairness and balance. Reacting to dynamic traffic changes, the server applies DBS to discover a new balanced $k$-position in light of $\Phi(x)$. Accordingly, channel allocation and item classification are adjusted to sustain the appropriateness.

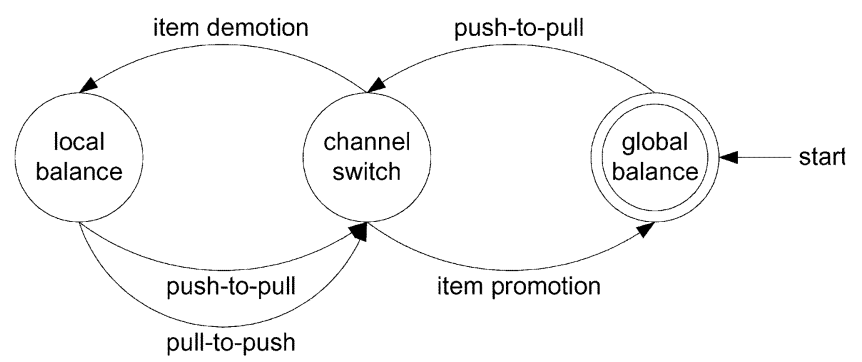

Fig. 4. State diagram in the deterministic balance search.

2) DBS Procedure: Fig. 4 shows the finite-state automata of the DBS procedure. Given $c$ available channels, the search domain $S=\left\{s_{1}, s_{2}, \ldots, s_{i}, \ldots, s_{c}\right\}$ includes $c$ local balances where $s_{i}$ presents $\left\langle c_{\text {pull }}, c_{\text {push }}, \overline{t_{\text {pull }}^{i}}, \overline{t_{\text {push }}^{i}}, \rho_{i}\right\rangle$. Consider that $\overline{t_{\text {pull }}}$ is vulnerable to traffic intensity and that $\overline{t_{\text {push }}}$ has a linear progression with the amount of push items. Two criteria are used to conduct the search process below.

Criterion 1: The pull data delivery must have a stable traffic intensity $(\rho<1)$.

Criterion 2: $\overline{t_{\text {pull }}^{i}} \leq \overline{t_{\text {push }}^{i}}$ is imperative at any possible local balance $s_{i}$.

Imbalance States: Starting at the initial state where all channels are in the push mode, the DBS procedure intentionally presumes an occurrence of imbalance state, as follows:

1) one more push-to-pull or pull-to-push channel switch will lead to a lower $\overline{t_{\text {push }}}$;

2) one more pull-to-push channel switch will not violate the stable restriction $\rho<1$.

An imbalance will trigger a trial if a push-to-pull channel switch leads to a new local balance where $\overline{t_{\text {push }}}$ is lower than that in the last state. Explicitly, an additional pull channel will cause a larger $\overline{t_{\text {push }}}$ and a lower $\overline{t_{\text {pull }}}$ vice versa. Therefore, the search process will repeatedly demote an item $d_{i}$ of the minimal $\Phi(i)$ in $U^{h}$ into $U^{c}$. The repetition of item demotion will stop as long as one more item demotion will violate criterion 1 or 2 .

Stop Conditions: Whereas an increase of $\overline{t_{\text {push }}}$ means a decrease of $\overline{t_{\text {pull }}}$, there exists a cross point where the search process reaches a local balance, as defined in Definition 2. To look for a global balance, as defined in Definition 3, the search process will repeat the channel switch until it meets one of the following stop conditions:

1) one more push-to-pull or pull-to-push channel switch will result in a larger $\overline{t_{\text {push }}}$;

2) one more pull-to-push channel switch will make the pull traffic intensity unstable as $(\rho \geq 1)$;

3) all channels are in the pull mode.

Definition 2: A local balance is a state where $\overline{t_{\text {push }}}$ is the minimum and also the traffic intensity is stable in response to a pull-to-push or push-to-pull channel switch.

Definition 3: A global balance is a state where $\overline{t_{\text {push }}}$ is a global minimum and, also, the traffic intensity is stable.

During the search for the global balance in $S$, the trial of the push-to-pull channel switch is repeated if a trial results in a lower $\overline{t_{\text {push }}^{i}}$. If no push channel is available, that is, in a pure pull-based mode, $\overline{t_{\text {pull }}}$ will alternatively be used to compare with the last $\overline{t_{\text {push }}}$. It is noted that when a push-to-pull channel switch 
TABLE II

SIMULATION PARAMETERS DESCRIPTION

\begin{tabular}{cll}
\hline term & \multicolumn{1}{c}{ meaning } & \multicolumn{1}{c}{ value } \\
\hline$m$ & the number of items in a broadcast databast $D$ & $100,1000,10000$ \\
$c$ & the number of downward channels (channel partition) & $10,50,100$ \\
$b$ & channel bandwidth & $100,500,1000$ KBytes \\
$\lambda$ & access rate in a time unit (workload) & variable $10 \sim 100$ \\
$\theta$ & skew coefficient in the Zipf distribution (access pattern) & variable $-2.0 \sim 2.0$ \\
$\mu$ & channel service rate $b / s$ & variable \\
$\omega$ & mean client patience & $100,1000,10000$ \\
\hline
\end{tabular}

causes an upward $\overline{t_{\text {push }}^{i}}$, the search will reflectively learn that $s_{i+1}$ in the last trial is the global balance. Then, a pull-to-push channel switch is employed backward. Only if not violating criteria 1 and 2 will the search process repeatedly promote an item $d_{i}$ of the maximal $\Phi(i)$ in $U^{c}$ into $U^{h}$. At the global balance, the number of items in $U^{h}$ is given to represent the $k$ position where the cutoff point splits $\Phi(x)$. Meanwhile, the partition of channels is the most effective. Consequently, the server is able to perform periodically the deterministic balance search to adjust channel allocation and item classification with respect to dynamic traffic changes, thereby maintaining the reliability and efficiency for the hybrid data delivery in a multichannel data broadcast environment.

\section{Performance Evaluation}

Section V-A models the simulation environment, Section V-B assesses the accuracy of the MCTA technique, and Section V-C evaluates the efficacy of the DBS technique. The experimental remarks and discussion are summarized in Section V-D.

\section{A. Simulation Environment}

Dynamic Traffic Generation: Table II lists the simulation parameter description. We generate the discrete access frequency distribution by the Zipf distribution [37], expressed as $\lambda_{i}=\lambda \cdot\left((1 / i)^{\theta}\right) /\left(\sum_{1 \leq i \leq m}(1 / i)^{\theta}\right)$, with the skew coefficient $\theta$ and workload $\lambda=\sum_{1 \leq i \leq m} \lambda_{i}$. The Zipf distribution is used to model the nonuniform discrete distribution. The distribution is more skewed as $\theta$ increases, but becomes uniform as $\theta=0$. We vary the values of $\lambda$ and $\theta$ to generate a new access frequency distribution per broadcast cycle.

Evaluation Measure: Regarding the MCTA procedure, three reflective bases are inspected in this simulation: the first item $\left(\mathrm{d}_{\mathrm{FI}}\right)$, the middle item $\left(\mathrm{d}_{\mathrm{MI}}\right)$, and the last item $\left(\mathrm{d}_{\mathrm{LI}}\right)$ in each $P_{x}$. We generate four access frequency distributions, $\Phi_{\mathrm{FI}}(x), \Phi_{\mathrm{MI}}(x), \Phi_{\mathrm{LI}}(x)$, and $\Phi_{\Delta}(x)$, by using $\mathrm{d}_{\mathrm{FI}}, \mathrm{d}_{\mathrm{MI}}, \mathrm{d}_{\mathrm{LI}}$ and the trigonometric tuning method. Then, we examine the accuracy differences of $\Phi_{\mathrm{FI}}(x), \Phi_{\mathrm{MI}}(x), \Phi_{\mathrm{LI}}(x)$ and $\Phi_{\Delta}(x)$ in comparison with $\Psi(x)$. To simplify demonstration, the same reflective base is taken in all $P_{x} s$. The DBS procedure discovers the cutoff points in $\Phi_{\mathrm{FI}}(x), \Phi_{\mathrm{MI}}(x), \Phi_{\mathrm{LI}}(x)$, and $\Phi_{\Delta}(x)$ for optimizing channel allocation and item classification. In terms of time difference, the measured $\overline{t_{\mathrm{push}}}$ is compared with the optimum.

- MCTA accuracy difference: Let $\Lambda_{i}$ be the difference between the estimated and accurate $\lambda_{i} s$ of an item $d_{i}$. Given with a flat broadcast program $P$, the mean $\Lambda$ is $\mu=$
$E(\Lambda)=\sum_{1<i<m}\left(\Lambda_{i}\right) /(m)$ and the standard deviation is $\sigma=\sqrt{\left(\sum_{1 \leq i \leq m}\left(\Lambda_{i}-\mu\right)^{2}\right) /(m)}$.

- DBS time difference: The time difference between the measured $\min \overline{t_{\text {push }}}$ based on $\Phi(x)$ and the optimal $\min \overline{t_{\text {push }}}$ based on $\Psi(x)$ presents the quality of the DBS technique.

\section{B. MCTA Performance Evaluation}

This section shows the estimations by using the MCTA technique under dynamic workload and skew access pattern. In addition, we investigate the MCTA technique in terms of sensitivities to dynamic workload, access pattern, channel partition, and the joint changes. The sensitivity to client patience was examined in [14].

1) MCTA Under Dynamic Workload: It is visible in Fig. 5 that $\Phi_{\mathrm{FI}}^{x}(x)$ and $\Phi_{\mathrm{MI}}^{x}(x)$ are "wave-like." The concatenation of $\Phi^{x}(x)$ s results in $\Phi(x)$. Observe that $\Psi(x)$ is bounded by $\Phi_{\mathrm{FI}}(x)$ and $\Phi_{\mathrm{MI}}(x)$. In Fig. 5(a), when the workload increases, $\Phi_{\mathrm{FI}}^{x}(x)$ is slightly above $\Psi(x)$, but $\Phi_{\mathrm{MI}}^{x}(x)$ is below $\Psi(x)$. However, the positions of $\Phi_{\mathrm{FI}}^{x}(x)$ and $\Phi_{\mathrm{MI}}^{x}(x)$ are reverse under the decreasing workload. $\Phi_{\mathrm{FI}}^{x}(x)$ is convex with a peak at $d_{\mathrm{MI}}$ as the workload increases; comparatively, $\Phi_{\mathrm{FI}}^{x}(x)$ is concave and $d_{\mathrm{MI}}$ becomes a foot as workload decreases. In contrast, $\Phi_{\mathrm{MI}}^{x}(x)$ is a mirror rendering corresponding to $\Phi_{\mathrm{FI}}^{x}(x)$ below $\Psi(x)$. Finally, it is prominent that $\Phi_{\Delta}^{x}(x)$ is very close to $\Psi(x)$.

The previously discussed phenomena are explained as follows. Depending on the reflective base in (5), the larger the value of $\gamma_{j}^{x} / \gamma_{i}^{x}$, the larger the value of $\lambda_{j}^{x}$ is. Because $d_{\mathrm{LI}}^{x}$ is just the item ahead of $d_{\mathrm{FI}}^{x}$ cyclically, $d_{\mathrm{FI}}^{x}$ and $d_{\mathrm{LI}}^{x}$ face the same situation. The situation is symmetric with $d_{\mathrm{MI}}^{x}$ as the symmetric point. If an item is scheduled earlier within the first half of $P_{x}$, the rate of its push access to impatient requests can be more than those of other items scheduled lately. Hence, the estimated $\lambda_{j}^{x}$ of $d_{j}^{x}$ can be higher than the real value when $d_{\mathrm{FI}}^{x}$, the item of the largest $\lambda_{i}^{x} / \gamma_{i}^{x}$, serves as the reflective base. Likewise, using $d_{\mathrm{MI}}^{x}$ whose $\lambda_{i}^{x} / \gamma_{i}^{x}$ is the smallest as the reflective base can cause $\Phi_{\mathrm{MI}}^{x}(x)$ to be lower than $\Psi(x)$. In the case of a decreasing workload, $\Phi_{\mathrm{FI}}^{x}(x)$ and $\Phi_{\mathrm{MI}}^{x}(x)$ in Fig. 5(b) are the inverted reflections of the corresponding curves in Fig. 5(a). This is because the rate of $\lambda_{\mathrm{FI}}^{L}$ to $\gamma_{\mathrm{FI}}^{L}$ is the smallest in a broadcast cycle and, thus, the estimated access frequency for another item is lower than its real value.

2) MCTA Under Dynamic Access Pattern: There are several observations presented. First, as depicted in Fig. 6(a), a hybrid data delivery model is profitable in the case of a higher skew pattern. Using parts of channels to broadcast fewer items of relatively high access frequency can reduce the mean access time. 


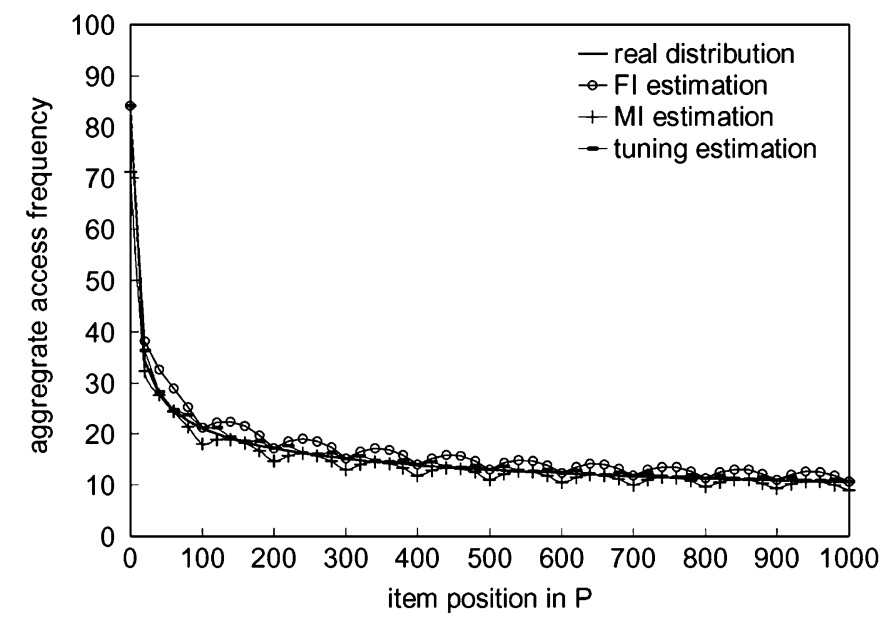

(a)

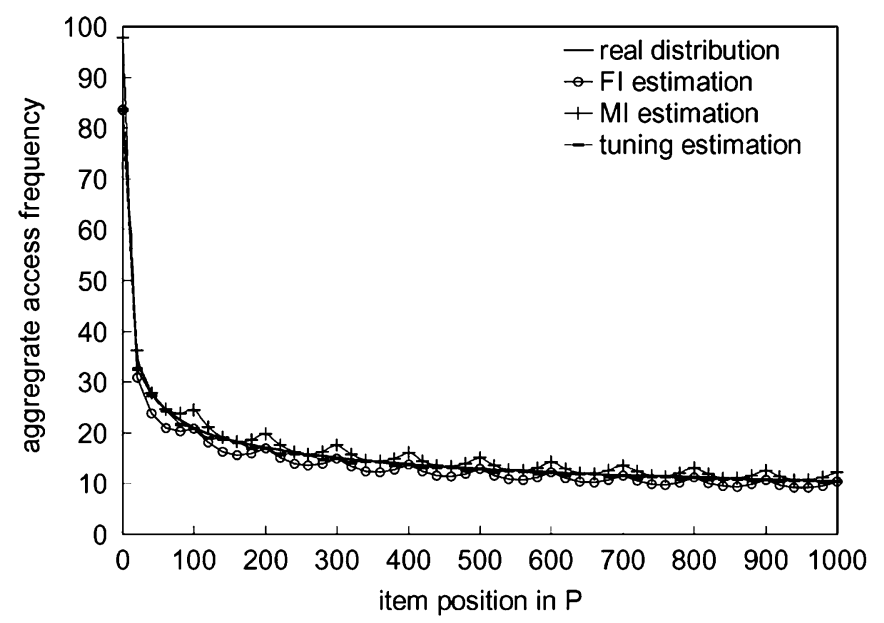

(b)

Fig. 5. MCTA under dynamic workload (baseline: $m=1000, c=10, \theta=0.3$, and $\lambda=100 \leftrightarrow 200$ ). (a) Increasing workload. (b) Decreasing workload.

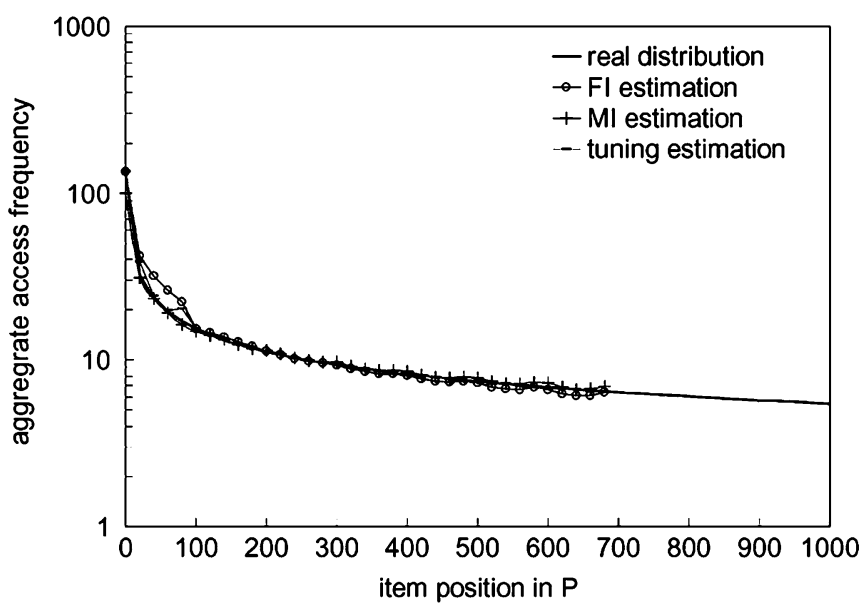

(a)

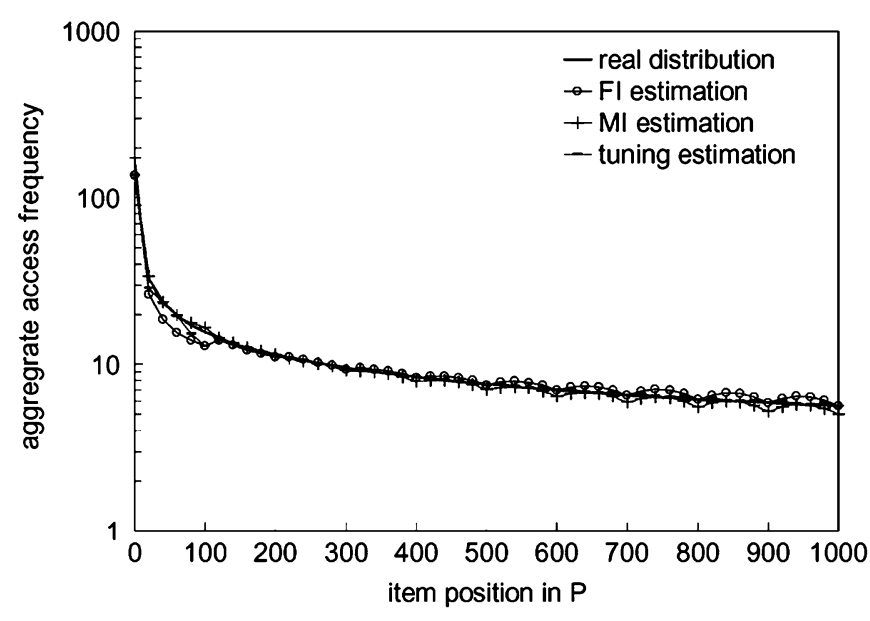

(b)

Fig. 6. MCTA under dynamic access pattern (baseline: $m=1000, c=10, \lambda=100$, and $\theta=0.3 \leftrightarrow 0.6$ ). (a) Increasing skew pattern. (b) Decreasing skew pattern.

Oppositely, as shown in Fig. 6(b), a pure push delivery is beneficial under a less skew access pattern. Second, the MCTA technique has high accuracy except when an item of a drastic change of access frequency is selected as the reflective base. In the context where item access frequencies change drastically, it is suggested that $d_{\mathrm{MI}}$ is more suitable to avoid performance degradation. Third, the effect of the trigonometric method is prominent. $\Phi_{\Delta}(x)$ is relatively closer to $\Psi(x)$ than $\Phi_{\mathrm{FI}}(x)$ and $\Phi_{\mathrm{MI}}(x)$ are. Consequently, the MCTA technique with the trigonometric tuning method is able to attain very high accuracy.

3) Sensitivity to Channel Variation: As shown in Fig. 7(a), the curves of $\Phi_{\mathrm{FI}}(x), \Phi_{\mathrm{MI}}(x)$, and $\Phi_{\Delta}(x)$ go up slightly corresponding to the increase of channel number labeled in the $x$-axis. However, the standard deviations of accuracy difference go down. For example, the difference percents of $\Phi_{\Delta}(x)$ are $4.57 \%, 4.98 \%, 5.14 \%, 5.22 \%, 5.28 \%$, and $5.32 \%$, but their deviations are $3.06,0.70,0.38,0.18,0.12$, and 0.09 . This is because the gradient of item classification is coarse in the case of fewer channels and can be refined with more channels. The MCTA technique can attain a higher accuracy in a small $P_{x}$ because the access frequencies of these items are close. Although the fast increase of workload can cause some items having drastic changes of access frequencies, the low accuracy difference as depicted in Fig. 7(a) presents the robustness of the MCTA technique to the variance of channel number. Note that the case of a light workload is omitted, because the server has the exact access frequencies of most items delivered in the pull mode.

4) Sensitivity to Workload Variation: Fig. 7(b) depicts the scalable performance of the MCTA technique. The accuracy difference increases slowly corresponding to the incremental workload change labeled in the $x$-axis. We observe that an increasing difference ascribes to the drastic change of workload in few $P_{x} \mathrm{~s}$, which contain relatively hotter items. Selecting a reflective base with a drastic change of access frequency will increase the accuracy difference. It is noteworthy that the accuracy reduction is confined to a few $P_{x}$ s; hence, the MCTA technique is still robust. This experimental results in essence conform to the general agreement that data broadcast is beneficial to the scalability problem.

5) Sensitivity to Access Pattern Variation: As depicted in Fig. 7(c), the less drastic the access pattern changes, the higher accuracy the MCTA technique has. It is interesting to find that $\Phi_{\mathrm{MI}}(x)$ is closer to $\Psi(x)$ than others when $\theta$ varies from 0 to a higher value. Explicitly, the items near the head and the rare of 


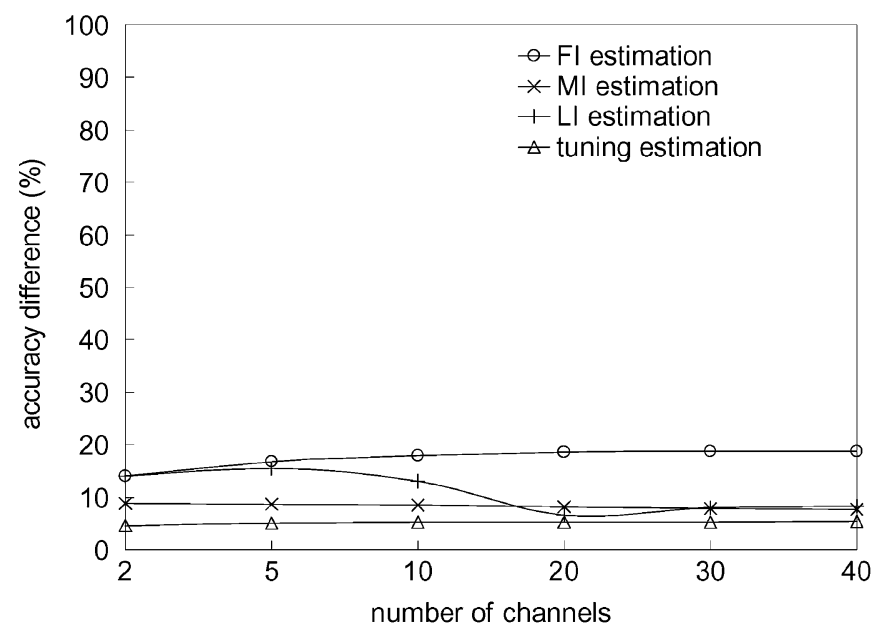

(a)

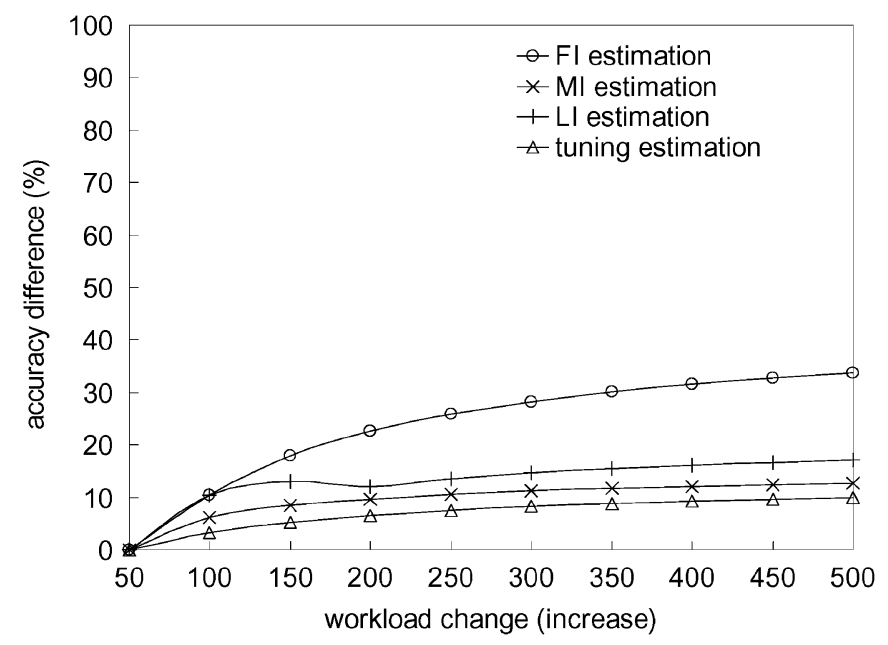

(b)

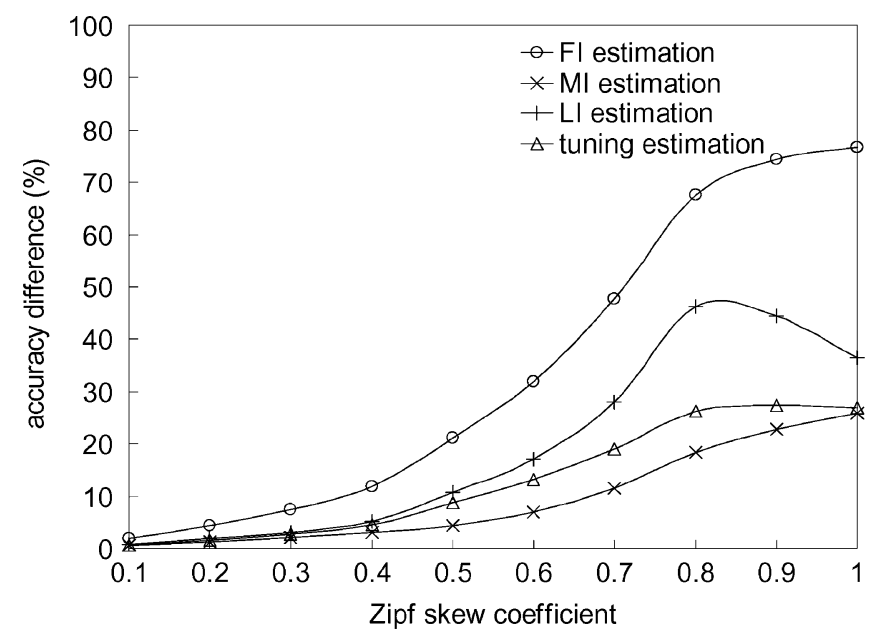

(c)

Fig. 7. Sensitivity of MCTA to traffic factors. (a) $m=1000, c \cdot \mu=100, \theta=$ $0.5, \lambda=50 \leftrightarrow 150$, channel variation. (b) $m=1000, c \cdot \mu=100, \theta=$ $0.5, \lambda=50 \leftrightarrow 500$, workload variation. (c) $m=1000, c \cdot \mu=100, \lambda=$ $150, \theta=0 \leftrightarrow 1.0$, access pattern variation.

each $P_{x}$, such as $d_{\mathrm{FI}}$ or $d_{\mathrm{LI}}$, can have higher variations in access frequency. Thus, $\Phi_{\mathrm{FI}}(x)$ affects the performance of the trigonometric tune poorly. Comparatively, $d_{\mathrm{MI}}$ is less susceptible in this context where the access pattern has a drastic change. In addition, the curves go up smoothly or downward, especially while the access pattern becomes very skewed ( $\theta$ from 0 to $\geq 0.8$ ). As measured, most workload focuses on a few items of very high access frequencies. The estimated frequencies of these items account for most of accuracy difference. Oppositely, other items have lower estimation difference.

6) Sensitivity to Joint Variation: Table III lists the numerical results of the difference percent and standard deviation. The workload $\lambda=200 \leftrightarrow 300$ initializes most items in the push mode. Note that the effect of the MCTA technique is scaled up to the data size. When the same access pattern is examined under $m=1000$ and $m=1000 \times 10$, the accuracy differences rise no more than $10 \%$ in all cases and the difference deviations decrease. For example, in the case of $c=\mu=10, \lambda=$ $200 \rightarrow 300$, and $\theta=0.5 \rightarrow 1, \Phi_{\Delta}(x)$ 's difference is $12.71 \%$ as $m=1000$ and 20.5 as $m=10000$; however, the respective difference deviations are 0.091 and 0.023 . In addition, there are several observations below. First, more channels can make the item classification more precise and improve the estimation accuracy. Second, an increasing/decreasing workload directly influences the accuracy difference to be large/small. The MCTA technique still attains high accuracy even though the workload is very heavy. Third, $\Phi_{\Delta}(x)$ has the best performance in most cases. Notwithstanding that $\Phi_{\mathrm{MI}}(x)$ is better in a few cases, $\Phi_{\Delta}(x)$ has similar accuracy to $\Phi_{\mathrm{MI}}(x)$. Finally, under a drastic change of access pattern, $\Phi_{\mathrm{MI}}(x)$ is more reliable except the case where the workload fast increases. Consequently, the MCTA technique with a reliable reflective base and the trigonometric tuning method is able to attain better performance.

\section{AMD Performance Evaluation}

In this section, the comparisons between the DBS technique with MCTA estimation, i.e., $\Phi(x)$, and the DBS technique with the real traffic, i.e., $\Psi(x)$, are evaluated in terms of sensitivities to dynamic workload, access pattern, and channel variations. Note that the AMD mechanism is evaluated under heavy traffic $(\rho \geq 1)$. We omit the case of light traffic $(\rho<1)$ because the server can adopt an exclusive pull delivery without the need of MCTA estimation.

1) Sensitivity to Dynamic Workload: Fig. 8 instantiates the procedure of the AMD mechanism, which iteratively searches for the global balance point when dynamic workload changes from 50 to $100 \leftrightarrow 500$ within a cycle time. The measures of mean access time, channel allocation, and item classification are depicted in Fig. 8(a); the inner histograms present the number of push items. With the cyclic MCTA estimation $\Phi(x)$, the DBS procedure reacts to a workload change. As illustrated in Fig. 8(a), the data delivery is be adapted in the exclusive pull, hybrid, or exclusive push model.

In addition, Fig. 8(b) depicts the comparison between $\overline{t_{\text {push }}}$ and the optimum obtained by using the DBS procedure with $\Phi(x)$ and $\Psi(x)$. Observe that the small time difference shows the feasibility of the AMD mechanism. Namely, the MCTA accuracy difference, as mentioned in Section V-B, just slightly affects the performance. Significantly, we find in Fig. 8(b) that the small time difference mainly occurs when the server employs a hybrid data delivery. In the exclusive push delivery, all items 
TABLE III

ACCURACY MEASUREMENT: $\Phi_{\mathrm{FI}}(x), \Phi_{\mathrm{MI}}(x), \Phi_{\mathrm{LI}}(x), \operatorname{AND} \Phi_{\mathrm{FI}}(x)$ VERSUS $\Psi(x)$

\begin{tabular}{rrrrrrrrrrrrrrrc}
\hline & item & \multicolumn{1}{c}{ workload $\lambda$} & pattern $\theta$ & \multicolumn{4}{c}{$\Phi(x)$ difference $\Lambda \%$} & \multicolumn{4}{c}{$\Phi(x)$ difference deviation } \\
\hline$c$ & $\mu$ & \multicolumn{1}{c}{$m$} & from & to & from & to & $\mathrm{d}_{\text {FI }}$ & \multicolumn{1}{c}{$\mathrm{d}_{\text {MI }}$} & \multicolumn{1}{c}{$\mathrm{d}_{\mathrm{LI}}$} & \multicolumn{1}{c}{$\Delta$} & \multicolumn{1}{c}{$\mathrm{d}_{\mathrm{FI}}$} & $\mathrm{d}_{\mathrm{MI}}$ & \multicolumn{1}{c}{$\mathrm{d}_{\mathrm{LI}}$} & $\Delta$ \\
\hline 10 & 10 & 1000 & 200 & 300 & 0.5 & 1.0 & 26.81 & 11.98 & 13.92 & 12.71 & 0.193 & 0.086 & 0.099 & 0.091 \\
10 & 10 & 1000 & 200 & 300 & 1.0 & 0.5 & 18.15 & 7.67 & 9.55 & 7.07 & 0.045 & 0.019 & 0.023 & 0.017 \\
10 & 10 & 1000 & 300 & 200 & 0.5 & 1.0 & 17.60 & 8.17 & 8.50 & 8.19 & 0.255 & 0.118 & 0.123 & 0.118 \\
10 & 10 & 1000 & 300 & 200 & 1.0 & 0.5 & 12.90 & 9.69 & 7.73 & 5.73 & 0.032 & 0.024 & 0.019 & 0.014 \\
10 & 10 & 10000 & 200 & 300 & 0.5 & 1.0 & 37.63 & 15.43 & 27.91 & 20.50 & 0.057 & 0.020 & 0.037 & 0.023 \\
10 & 10 & 10000 & 200 & 300 & 1.0 & 0.5 & 16.35 & 6.87 & 12.85 & 7.31 & 0.004 & 0.002 & 0.003 & 0.002 \\
10 & 10 & 10000 & 300 & 200 & 0.5 & 1.0 & 32.78 & 13.60 & 25.09 & 16.80 & 0.131 & 0.055 & 0.100 & 0.067 \\
10 & 10 & 10000 & 300 & 200 & 1.0 & 0.5 & 11.86 & 8.38 & 9.21 & 6.02 & 0.003 & 0.002 & 0.002 & 0.002 \\
25 & 4 & 1000 & 200 & 300 & 0.5 & 1.0 & 22.50 & 11.01 & 10.76 & 8.95 & 0.164 & 0.080 & 0.078 & 0.065 \\
25 & 4 & 1000 & 200 & 300 & 1.0 & 0.5 & 17.67 & 8.08 & 7.81 & 5.98 & 0.014 & 0.020 & 0.019 & 0.015 \\
25 & 4 & 1000 & 300 & 200 & 0.5 & 1.0 & 13.78 & 7.78 & 7.23 & 5.20 & 0.212 & 0.120 & 0.111 & 0.080 \\
25 & 4 & 1000 & 300 & 200 & 1.0 & 0.5 & 12.03 & 9.64 & 8.86 & 4.51 & 0.029 & 0.023 & 0.022 & 0.011 \\
25 & 4 & 10000 & 200 & 300 & 0.5 & 1.0 & 33.25 & 15.20 & 16.53 & 15.74 & 0.043 & 0.019 & 0.021 & 0.020 \\
25 & 4 & 10000 & 200 & 300 & 1.0 & 0.5 & 19.22 & 9.06 & 11.58 & 7.39 & 0.004 & 0.002 & 0.002 & 0.002 \\
25 & 4 & 10000 & 300 & 200 & 0.5 & 1.0 & 27.07 & 12.92 & 11.79 & 11.82 & 0.109 & 0.052 & 0.047 & 0.047 \\
25 & 4 & 10000 & 300 & 200 & 1.0 & 0.5 & 12.98 & 10.73 & 8.47 & 5.81 & 0.003 & 0.002 & 0.002 & 0.001 \\
\hline
\end{tabular}

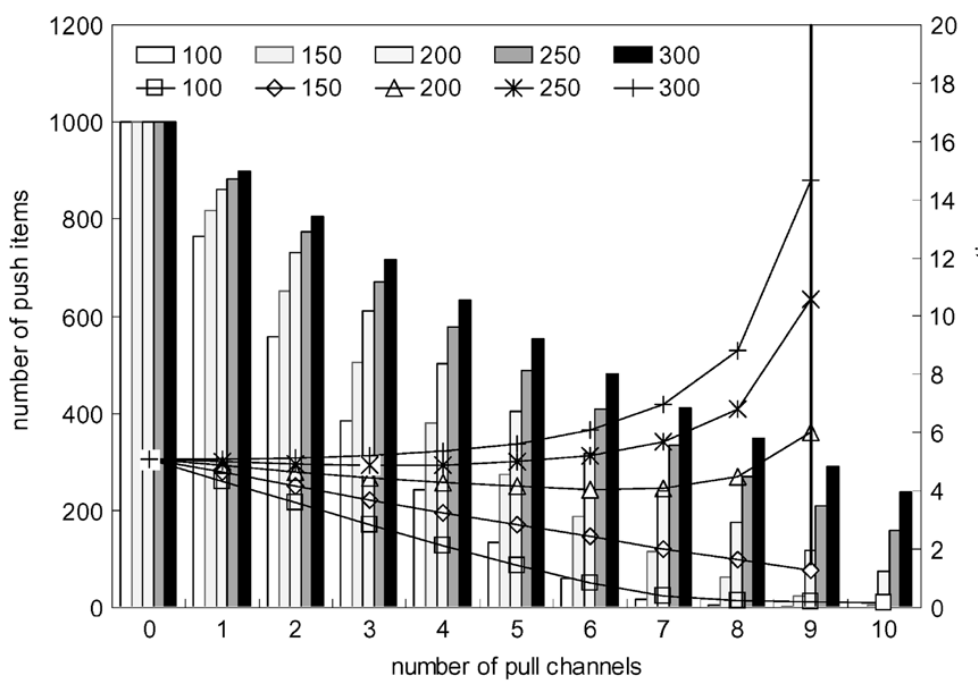

(a)

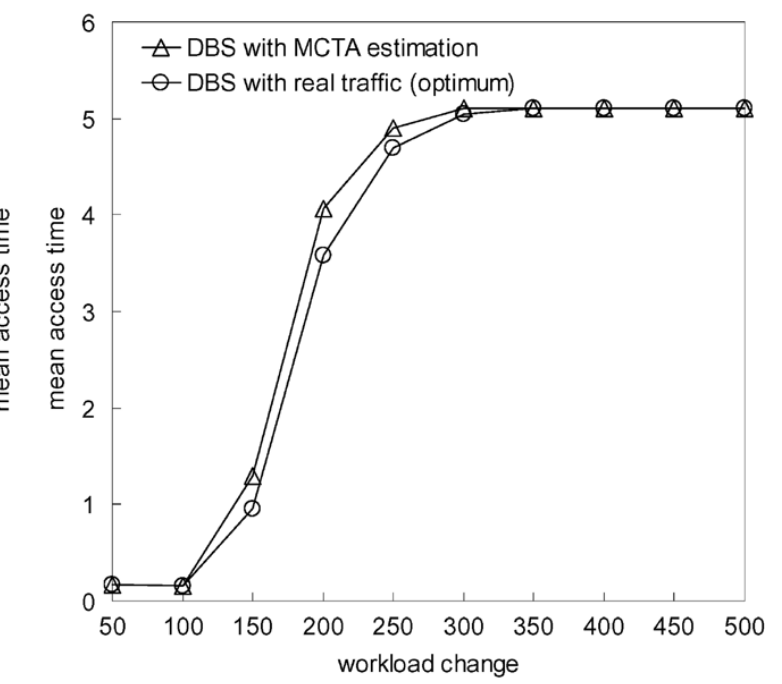

(b)

Fig. 8. Sensitivity to dynamic workload. (a) Balance point, DBS with MCTA estimation. (b) Balance comparsion, DBS with $\Phi(x)$ versus DBS with $\Psi(x)$ (baseline: $m=1000, c=10, \mu=10, \theta=0.5$ ).

are broadcast cyclically without respect to the access frequency. In contrast, the server need not perform the MCTA estimation in the exclusive pull delivery. Thus, it is rational to attribute the small time difference to the possible MCTA accuracy difference.

2) Sensitivity to Dynamic Access Pattern: Fig. 9 illustrates the sensitivity of the AMD mechanism against dynamic access pattern by an incremental value of the Zipf skew coefficient $\theta$ varying from 0 to $0.1 \leftrightarrow 1.4$ within a cycle time. We obtain several experimental results below. First, the skewer the access pattern, the lower $\overline{t_{\text {push }}}$ can be in the hybrid data delivery. Generally speaking, delivering items of high access frequency by the push mode will efficiently reduce the pull traffic load. As most workload belongs to part hot items, the number of these items decreases in response to the incremental value of $\theta$. Correspondingly, the number of channels used to convey these items decreases. Hence, the effect of the AMD mechanism agrees with the general consensus. Second, $\overline{t_{\text {push }}}$ in the case of dynamic access pattern is more susceptible than that in the case of dynamic workload, as mentioned before. Even under static workload, a change of access pattern may result in a higher $\overline{t_{\mathrm{push}}}$. Finally, it is notable that there is no difference between these two curves in Fig. 9(b). This phenomenon consequently shows the reliability of the AMD mechanism. That is, the DBS technique can carry out a reliable item classification and channel allocation in accordance with the MCTA estimation.

3) Sensitivity to Channel Allocation: Fig. 10 illustrates the sensitivity of the AMD mechanism to channel allocation under dynamic workload and dynamic access pattern. The numeral on top of a bar chart indicates the number of push channels to the total number of channels labeled in the $x$-axis. The experiment settings make the server apply a hybrid data delivery. The channel variation has influence on the item classification gradient. The precision of the gradient is improved by the increase of channel number, because each cluster contains fewer items whose access frequencies are similar.

We have shown in Section V-B.3 that the accuracy difference between $\Phi_{\Delta}(x)$ and $\Psi(x)$ is not expanded by the channel variation. As shown in Fig. 10(a), however, the difference be- 


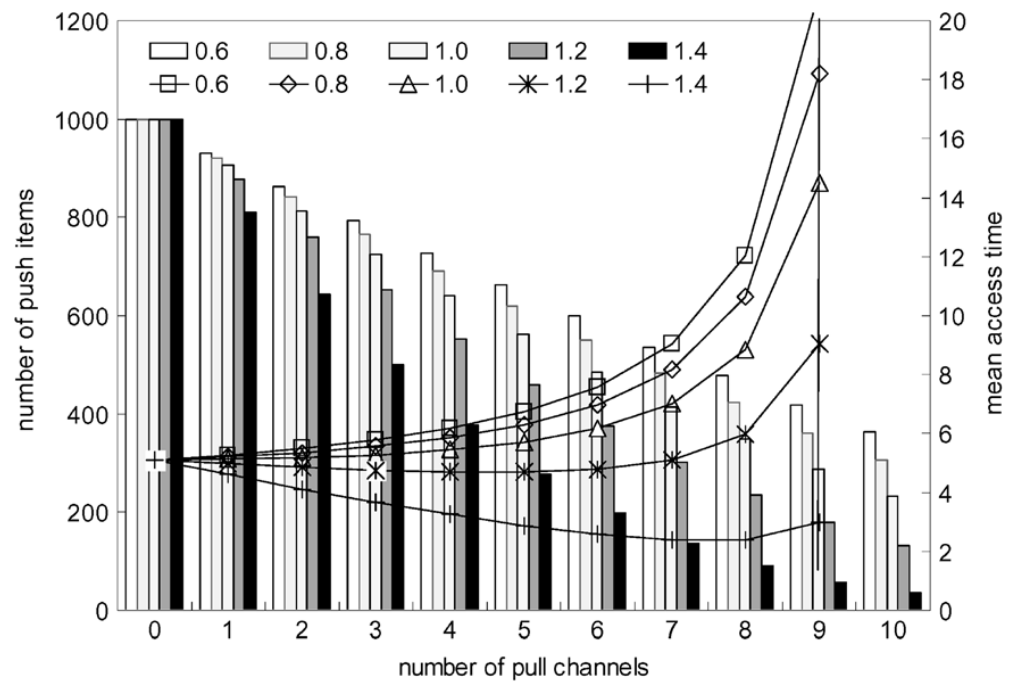

(a)

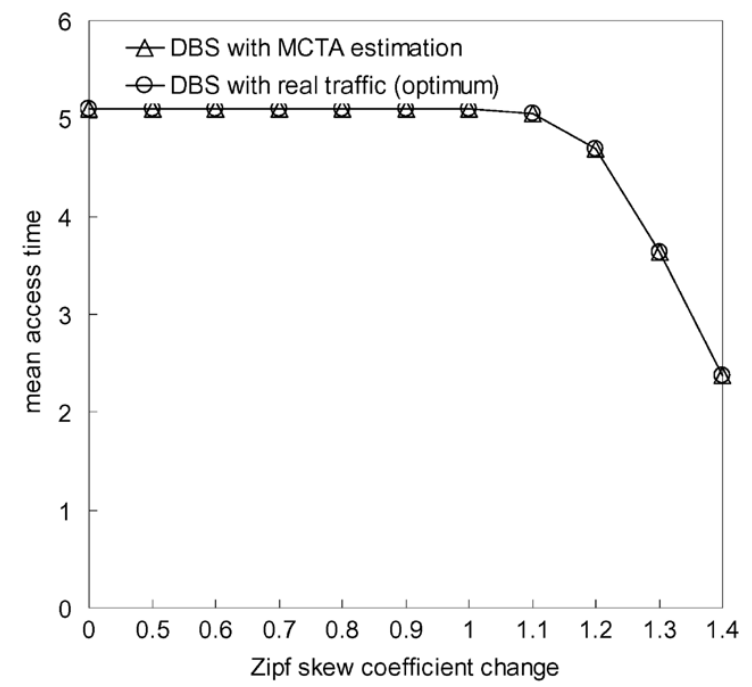

(b)

Fig. 9. Sensitivity to dynamic access pattern. (a) Balance point, DBS with MCTA. (b) Balance comparsion, DBS with $\Phi(x)$ versus DBS with $\Psi(x)$ (baseline: $m=1000, c=10, \mu=10, \lambda=200)$.

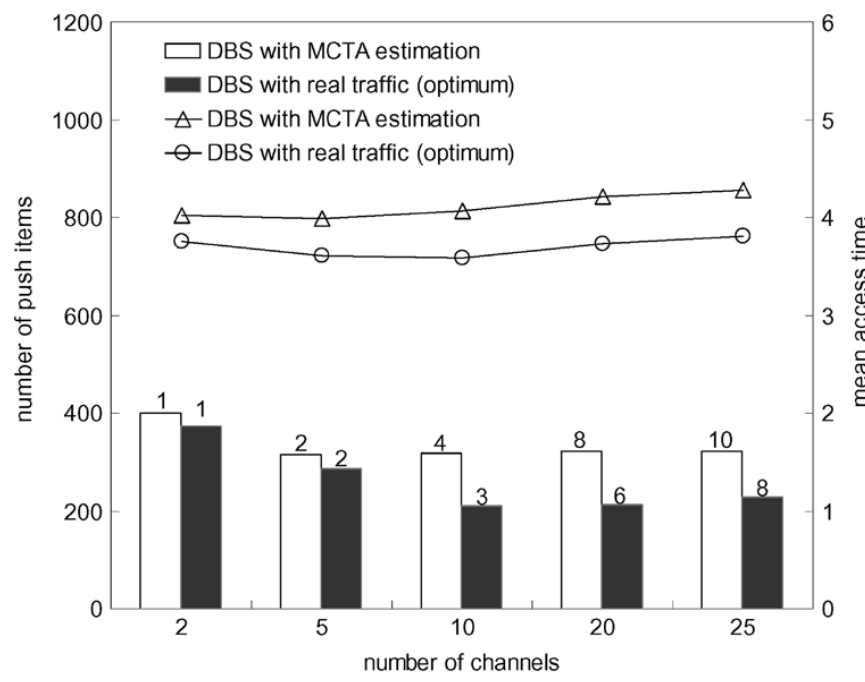

(a)

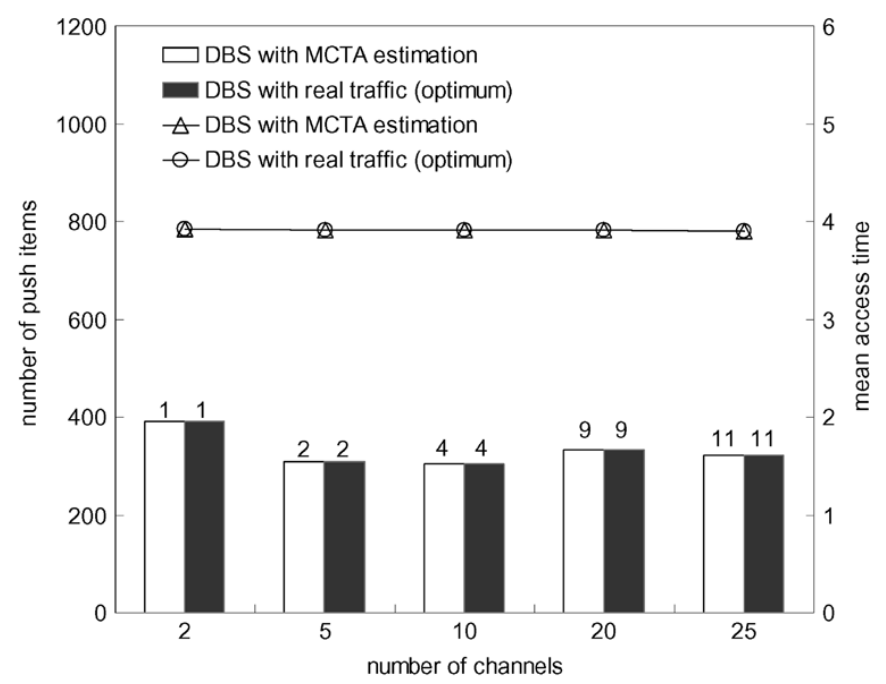

(b)

Fig. 10. Sensitivity to channel allocation. (a) Under dynamic workload, $\lambda=50 \rightarrow 200, \theta=0.5$. (b) Under dynamic access pattern, $\lambda=200, \theta=0.5 \rightarrow 1.0$ (baseline: $m=1000, c \cdot \mu=100$ ).

tween the measured $\overline{t_{\mathrm{push}}}$ and the optimum somewhat varies to some extent. In contrast, as depicted in Fig. 10(b), there is no time difference in some cases of channel variation versus dynamic access pattern. The above examinations attribute the time difference to the accuracy difference of MCTA estimation. As explained before in Section V-B, since the MCTA estimation is of high accuracy, we believe that the AMD mechanism is amenable.

\section{Summary and Discussion}

Extensive simulations have been conducted to investigate the design of the novel AMD mechanism. We have evaluated the MCTA and the DBS techniques against the performance impacts of traffic dynamics with several factors, including workload, skew access pattern, channel allocation, and joint changes.
In this section, the AMD mechanism has clearly demonstrated its adaptability for dynamic data broadcasting on multiple channels.

The MCTA technique is first examined in terms of accuracy difference. As shown by the experimental results, the MCTA estimation of access frequency distribution has low accuracy difference in comparison with the real one. The MCTA technique is scaled up to workload variation. Although it is more susceptible to drastic changes of the skew access pattern, more channel allocations can refine the gradient of item classification and, hence, improve the estimation accuracy. In addition, we have examined the relative performance of $\Phi_{\mathrm{FI}}(x), \Phi_{\mathrm{MI}}(x), \Phi_{\mathrm{LI}}(x)$, and $\Phi_{\Delta}(x)$ obtained with different reflective bases $d_{\mathrm{FI}}, d_{\mathrm{MI}}$, and $d_{\mathrm{LI}}$ and the trigonometric tuning method. It is shown that $\Phi_{\Delta}(x)$ has the best performance in general and that $\Phi_{\mathrm{MI}}(x)$ is similar to $\Phi_{\Delta}(x)$ at times. 
Therefore, in light of $\Phi_{\Delta}(x)$, we have conducted further experiments to investigate the DBS technique. Section V-C mentioned the significance of the DBS technique. By using the DBS technique, the data delivery is adapted in the exclusive pull, hybrid, or exclusive push model in response to dynamic traffic changes. The experimental results have shown that the DBS technique is not only able to minimize the push access and the pull response time, but also balance them. At the global balance was achieved, the item classification and channel allocation are very close to the optima. Consequently, the previous observations account for the feasibility and reliability of the AMD mechanism.

\section{CONCLUSION}

This paper has proposed a novel AMD mechanism, which supports both the MCTA and the DBS techniques, to pursue the fairness and robustness for a hybrid data delivery in multichannel data-dissemination environments. The contributions of this paper are two-fold. In response to dynamic traffic, the MCTA technique is able to periodically estimate the access frequencies of all items disseminated in the push channels. Furthermore, in light of the MCTA estimation, the DBS procedure employs a heuristic search for the global balance in which the push access time and the pull response time are minimized; meanwhile, the item classification and channel allocation are optimized. Extensive simulations have been conducted to evaluate the MCTA and DBS techniques. The experimental results have shown that the estimated access frequency distribution by using the MCTA technique has high accuracy. In addition, the DBS technique is robust in response to slight accuracy difference and notedly attains a global balance very close to the optimum. Consequently, the simulation results has shown the feasibility and reliability of the AMD mechanism under dynamic traffic changes.

\section{REFERENCES}

[1] S. Acharya, R. Alonso, M. J. Franklin, and S. B. Zdonik, "Broadcast disks: Data management for asymmetric communications environments," in Proc. ACM Int. Conf. Management of Data, May 1995, pp. $199-210$

[2] S. Acharya, M. J. Franklin, and S. B. Zdonik, "Balancing push and pull for data broadcast," in Proc. ACM Int. Conf. Management of Data, May 1997, pp. 183-194.

[3] S. Acharya and S. Muthukrishnan, "Scheduling on-demand broadcasts: New metrics and algorithms," in Proc. ACM MobiCom'98, 1998, pp. $43-54$.

[4] D. Aksoy and M. Franklin, "Rxw: A scheduling approach for large-scale on-demand data broadcast," IEEE/ACM Trans. Networking, vol. 7, no. 6, pp. 846-860, Jun. 1999.

[5] M. H. Ammar and J. W. Wong, "The design of teletext broadcast cycles," Perform. Eval., vol. 5, no. 4, pp. 235-242, 1985.

[6] D. Barbará, "Mobile computing and database-A survey," IEEE Trans. Knowledge Data Eng., vol. 11, no. 1, pp. 108-117, Jan./Feb. 1999.

[7] G. Bolch, S. Greiner, H. de Meer, and K. S. Trivedi, Queueing Networks and Markov Chains: Modeling and Performance Evaluation with Computer Science Applications. New York: Wiley, 1998.

[8] J. Cai and K.-L. Tan, "Tuning integrated dissemination-based information systems," Data Knowledge Engineering , vol. 30, no. 1, pp. 1-21, Jan./Feb. 1999.
[9] M. J. Donahoo, M. H. Ammar, and E. W. Zegura, "Multiple-channel multicast scheduling for scalable bulk-data transport," in Proc. 19th IEEE Annu. Joint Conf. IEEE Computer and Communications Societies, Mar. 1999, pp. 847-855.

[10] J. Fernandez-Conde and K. Ramamritham, "Adaptive disseminatin of data in time-critical asymmetric communication environments," Mobile Networks Applicat., vol. 9, no. 5, pp. 491-505, 2004.

[11] Y. Guo, C. M. Pinotti, and S. K. Das, "A new hybrid broadcast scheduling algorithm for asymmetirc communication systems," Mobile Networks Applicat., vol. 5, no. 3, pp. 39-51, 2001.

[12] S. Hameed and N. H. Vaidya, "Efficient algorithms for scheduling data broadcast," ACM/Baltzer Wireless Networks, vol. 5, no. 3, pp. 183-193, 1999.

[13] P. G. Hoel, S. C. Port, and C. J. Stone, Introduction to Probability Theory. Boston, MA: Houghton Mifflin, 1971.

[14] C.-L. Hu and M.-S. Chen, "Dynamic data broadcasting with traffic awareness," in Proc. 22nd IEEE Int. Conf. Distributed Computing Systems, Jul. 2002, pp. 112-119.

[15] J.-H. Hu, K. L. Yeung, G. Fend, and K. Leung, "A novel push-and-pull hybrid data broadcast scheme for wireless information networks," in Proc. IEEE Int. Conf. Communications, Jun. 2000, pp. 1778-1782.

[16] Q. Hu, D. L. Lee, and W.-C. Lee, "Dynamic data delivery in wireless communication environments," in Proceedings of ER'98 Workshops on Mobile Data Access. New York: Springer, 1998, vol. Lecture Notes Comp. Sci., 1552, pp. 218-229.

[17] J.-L. Huang and M.-S. Chen, "Broadcasting dependent data for ordered queries without replication in a multi-channel mobile environment," in Proc. 19th IEEE Int. Conf. Data Engineering, Mar. 2003, pp. 692-694.

[18] J.-L. Huang, W.-C. Peng, and M.-S. Chen, "Binary interpolation search for solution mapping on broadcast and on-demand channels in a mobile computing environment," in Proc. 10th ACM Int. Conf. Information and Knowledge Management, Nov. 2001, pp. 434-441.

[19] T. Imielinski and S. Viswanathan, "Adaptive wireless information systems," in Proc. ACM Special Interest Group on DataBase Systems, 1994, pp. 19-41.

[20] S. Jiang and N. H. Vaidya, "Scheduling data broadcast to "impatient" users," in Proc. ACM Int. Workshop Data Engineering for Wireless and Mobile Access, Aug. 1999, pp. 52-59.

[21] J. Jing, A. K. Elmargarmid, S. Helal, and R. Alonso, "Bit-sequences: A new cache invalidation method in mobile environments," ACM/Baltzer Mobile Networks Applicat., vol. 2, no. 2, pp. 115-127, 1997.

[22] K. Kleinrock, Queueing Systems, Volume I: Theory. New York: Wiley, 1975.

[23] W.-C. Lee, Q. Hu, and D. L. Lee, "A study on channel allocation for data dissemination in mobile computing environments," ACM/Baltzer Mobile Networks Applicat., vol. 4, no. 2, pp. 117-129, 1999.

[24] C.-W. Lin and D. L. Lee, "Adaptive data delivery in wireless communication environments," in Proc. 20th Int. Conf. Distributed Computing Systems, Apr. 2000, pp. 444-452.

[25] W.-C. Peng and M.-S. Chen, "Dynamic generation of data broadcasting programs for a broadcast disk array in a mobile computing environment," in Proc. 9th ACM Int. Conf. Information and Knowledge Management, Nov. 2000, pp. 38-45.

[26] K. Prabhakara, K. A. Hua, and J. Oh, "Multi-level multi-channel air cache designs for broadcasting in a mobile environment," in Proc. 16th IEEE Int. Conf. Data Engineering, Feb. 2000, pp. 167-176.

[27] K. Ramamritham, "Review-Multi-level multi-channel air cache designs for broadcasting in a mobile environment," ACM SIGMOD Dig. Rev., vol. 2, 2000.

[28] K. Stathatos, N. Roussopoulos, and J. S. Baras, "Adaptive data broadcast in hybrid networks," in Proc. 23rd Int. Conf. Very Large Data Bases, Aug. 1997, pp. 326-335.

[29] C.-J. Su, L. Tassiulas, and V. J. Tsotras, "Broadcast scheduling for information distribution," ACM/Baltzer Wireless Networks, vol. 5, no. 2, pp. 137-147, 1999.

[30] W. Sun, W. Shi, B. Shi, W. Ji, and Y. Yu, "A self-adaptive scheduling algorithm of on-demand broadcasts," in Proc. 4th ACM Int. Workshop Modeling, Analysis and Simulation of Wireless and Mobile System, Jul. 2001, pp. 139-145.

[31] K.-L. Tan and B. C. Ooi, Data Dissemination in Wireless Computing Environments. Boston, MA: Kluwer, 2000.

[32] K.-L. Tan and J. X. Yu, "A dynamic scheduler for the infinite air-cache," Data Knowledge Eng. , vol. 24, no. 1, pp. 97-112, 1997. 
[33] M. A. Weiss, Data Structures and Algorithm Analysis in C. Reading, MA: Addison Wesley, 1993.

[34] J. W. Wong, "Broadcast delivery," Proc. IEEE, vol. 76, no. 12, pp. 1566-1577, Dec. 1988.

[35] W. G. Yee and S. B. Navathe, "Efficient data access to multi-channel broadcast programs," in Proc. ACM Int. Conf. Information and Knowledge Management, Nov. 2003, pp. 153-160.

[36] J. X. Yu, T. Sakata, and K.-L. Tan, "Statistical estimation of access frequencies in data broadcasting environments," ACM/Baltzer Wireless Networks, vol. 22, no. 6, pp. 89-98, 2000

[37] G. K. Zipf, Human Behavior and the Principle of Least Effort. Reading, MA: Addison-Wesley, 1949.

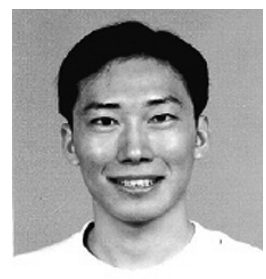

Chih-Lin Hu (S'02-M'04) received the B.S. degree in computer science from National Cheng-Chi University, Taipei, Taiwan, in 1997, the M.S. degree in computer science from National Chung-Hsin University, Taichung, Taiwan, in 1999, and the Ph.D. degree in electrical engineering from National Taiwan University, Taipei, Taiwan, in 2003.

He is currently a Researcher in BenQ Coproration, Taipei, Taiwan, where he joined the wireless function division, networking and communication group, in 2004. He was developing the protocol and system platforms for BenQ's mobile phones. Now he is a staff member in BenQ e-Home project, interoperating home network and mobile handheld devices across a home network. His research interests include broadcast information systems, mobile computing systems, and home networking technologies.

Dr. Hu received the best paper award in IEEE ICPADS' 00 .

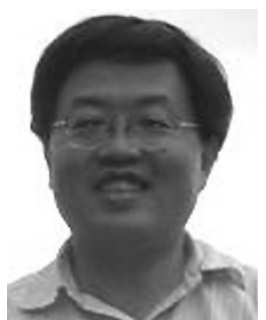

Ming-Syan Chen (S'88-M'88-SM'93-F'04) received the B.S. degree in electrical engineering from National Taiwan University, Taipei, Taiwan, and the M.S. and Ph.D. degrees in computer, information, and control engineering from The University of Michigan, Ann Arbor, in 1985 and 1988, respectively.

He was a Research Staff Member at IBM Thomas J. Watson Research Center, Yorktown Heights, NY, from 1988 to 1996 . He currently is a Professor and the Chairman of Graduate Institute of Communication Engineering and a Professor in the Electrical Engineering and the Computer Science and Information Engineering Departments, National Taiwan University. He holds, or has applied for, 18 U.S. patents and seven ROC patents in the areas of data mining, Web applications, interactive video playout, video server design, and concurrency and coherency control protocols. He has published more than 165 papers in his research areas, which include database systems, data mining, mobile computing systems, and multimedia networking.

Dr. Chen served as a Program Committee Member in many conferences and as an Associate Editor of IEEE TRANSACTIONS ON KNOWLEDGE AND DATA ENGINEERING from 1997 to 2001 and is currently on the Editorial Board of Very Large Data Base Journal, Knowledge and Information Systems Journal, Journal of Information Science and Engineering, and Journal of the Chinese Institute of Electrical Engineering. He was a Distinguished Visitor of the IEEE Computer Society for Asia-Pacific from 1998 to 2000. He served as the International Vice Chair for INFOCOM'05, Program Chair of Pacific Area Knowledge Discovery and Data Mining (PAKDD-02), Program Vice Chair of the International Conference on Parallel Processing (ICPP'03), Program Vice Chair of the Very Large Data Bases Conference (VLDB'02), General Chair of Real-Time Multimedia System Workshop in 2001, Program Chair of IEEE ICDCS Workshop on Knowledge Discovery and Data Mining in the World Wide Web in 2000, and Program Cochair of the International Conference on Mobile Data Management (MDM'03), International Computer Symposium (ICS) on Computer Networks, Internet and Multimedia in 1998 and 2000, and ICS on Databases and Software Engineering in 2002. He was a Keynote Speaker on Web data mining in the International Computer Congress in Hong Kong in 1999, a Tutorial Speaker on Web data mining in DASFAA'99 and on parallel databases in the 11th IEEE International Conference on Data Engineering in 1995 and also a Guest Coeditor for IEEE TKDE for a Special Issue on Data Mining in December 1996. He is a Recipient of the National Science Council (NSC) Distinguished Research Award and the K.-T. Li Research Penetration Award for his research work. He also received the Outstanding Innovation Award from IBM Corporation for his contribution to a major database product and received numerous awards for his research, teaching, inventions, and patent applications. He coauthored with his students for their works, which received the ACM SIGMOD Research Student Award and Acer Long-Term Thesis Awards. He is a Member of ACM. 